San Jose State University

SJSU ScholarWorks

Master's Theses

Master's Theses and Graduate Research

1990

\title{
Properties of evaporated Co/Pt multilayer thin films for optical recording
}

Hoa Do

San Jose State University

Follow this and additional works at: https://scholarworks.sjsu.edu/etd_theses

\section{Recommended Citation}

Do, Hoa, "Properties of evaporated Co/Pt multilayer thin films for optical recording" (1990). Master's Theses. 3255.

DOI: https://doi.org/10.31979/etd.pfnu-r6jn

https://scholarworks.sjsu.edu/etd_theses/3255

This Thesis is brought to you for free and open access by the Master's Theses and Graduate Research at SJSU ScholarWorks. It has been accepted for inclusion in Master's Theses by an authorized administrator of SJSU ScholarWorks. For more information, please contact scholarworks@sjsu.edu. 


\section{INFORMATION TO USERS}

The most advanced technology has been used to photograph and reproduce this manuscript from the microfilm master. UMI films the text directly from the original or copy submitted. Thus, some thesis and dissertation copies are in typewriter face, while others may be from any type of computer printer.

The quality of this reproduction is dependent upon the quality of the copy submitted. Broken or indistinct print, colored or poor quality illustrations and photographs, print bleedthrough, substandard margins, and improper alignment can adversely affect reproduction.

In the unlikely event that the author did not send UMI a complete manuscript and there are missing pages, these will be noted. Also, if unauthorized copyright material had to be removed, a note will indicate the deletion.

Oversize materials (e.g., maps, drawings, charts) are reproduced by sectioning the original, beginning at the upper left-hand corner and continuing from left to right in equal sections with small overlaps. Each original is also photographed in one exposure and is included in reduced form at the back of the book.

Photographs included in the original manuscript have been reproduced xerographically in this copy. Higher quality 6" $\times 9$ " black and white photographic prints are available for any photographs or illustrations appearing in this copy for an additional charge. Contact UMI directly to order.

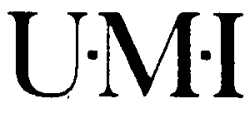

University Microtims International

A Bell \& Howell Intormation Company

300 North Zeeo Road. Ann Arbor. MI 48106-1346 LSA

$313761.4700 \quad 800521.0600$ 
Order Number 1340508

Properties of evaporated Co/Pt multilayer thin films for optical recording

$$
\text { Do, Hoa, M.S. }
$$

San Jose State University, 1990

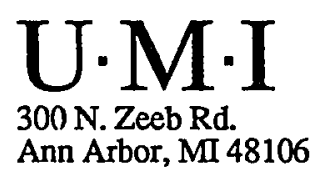




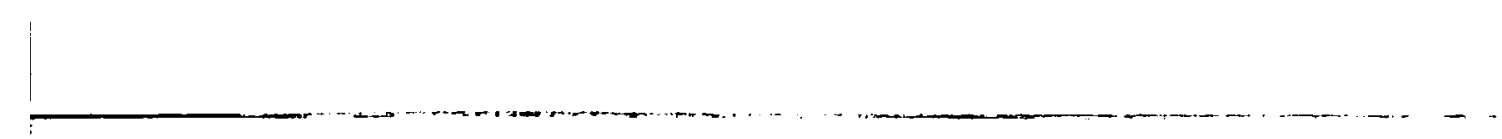




\title{
PROPERTIES \\ OF EVAPORATED CO/Pt \\ MULTILAYER THIN FILMS \\ FOR OPTICAI RECORDING
}

\begin{abstract}
A Thesis
Presented to

The Faculty of the Department of Materials Engineering San Jose State University
\end{abstract}

\author{
In Partial Fulfillment \\ of the Requirements for the Degree \\ Master of Sciences
}

By

HOA DO

May, 1990 
APPROVED FOR THE DEPARTMENT OF MATERIALS ENGINEERING
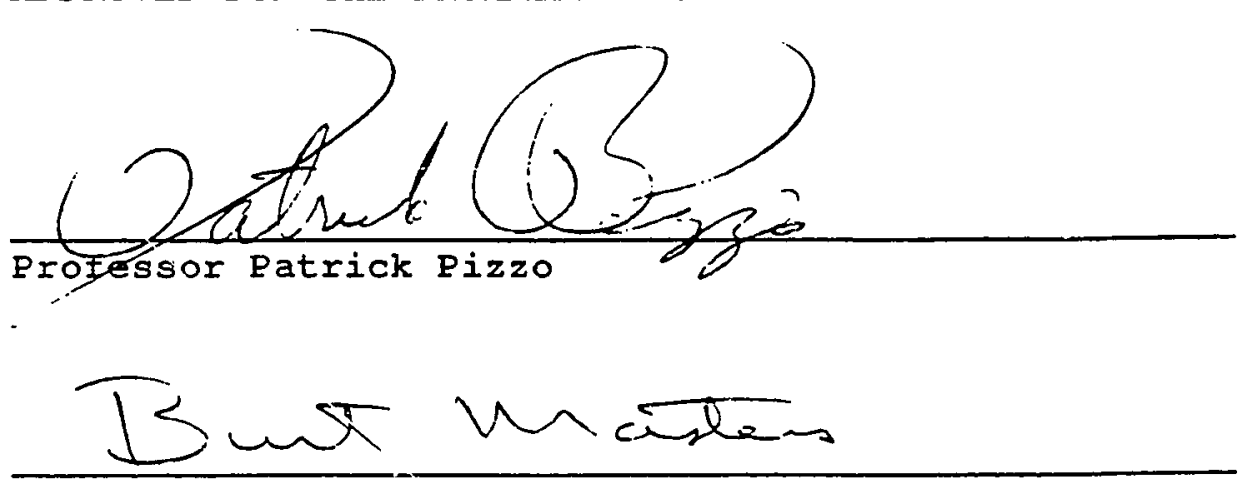

Professor Burt Masters

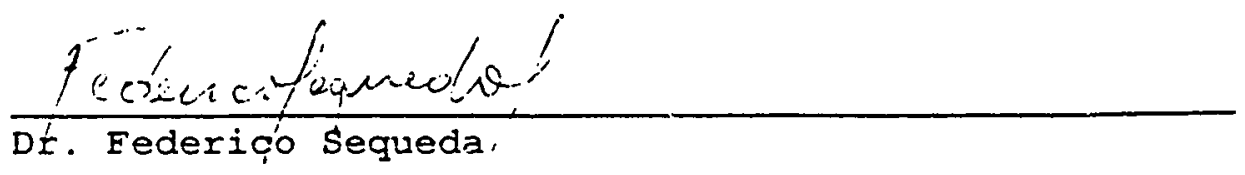

APPROVED FOR THE UNIVERSITY

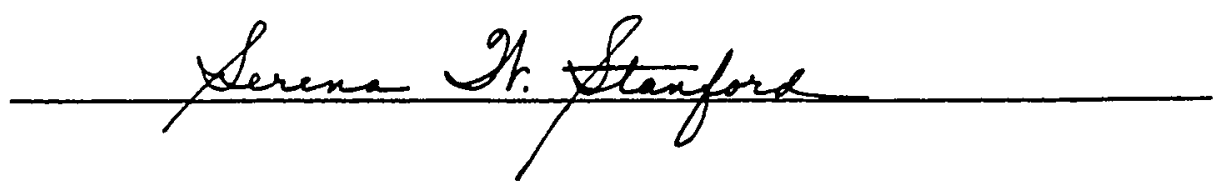




\author{
ABSTRACT \\ PROPERTIES OF EVAPORATED CO/PE MULTIIAAYER \\ THIN FILMS FOR OPTICAI RECORDING \\ By Hoa Do
}

Co/Pt ultra-thin multilayer films were deposited onto glass substrates by electron-beam evaporation from Co and Pt sources. The thickness of the Co and Pt layers was varied from $0.1 \mathrm{~nm}$ to $2 \mathrm{~nm}$ and from $0.3 \mathrm{~nm}$ to $2 \mathrm{~nm}$ respectively. The total film thickness was varied from $10 \mathrm{~nm}$ to $100 \mathrm{~nm}$. For Co/Pt multilayer films with a constant Pt layer thickness of $1 \mathrm{~nm}$, the remanent Kerr rotation is saturated at $\theta_{\mathrm{k}}=0.2^{\circ}$ for a Co layer thickness of $0.4 \mathrm{~nm}$ or less. The remanent Kerr rotation of Copt multilayer films with a constant Co layer thickness increases as the Pt thickness decreases. The magnetization and the Curie temperature of the multilayers increase with increasing co content of the film. Because of their excellent magnetic properties and their high corrosion and oxidation resistance, these co/Pt layered structures are very promising candidates for magneto-optic recording. 


\section{ACKNOWLEDGEMIHNTS}

I am grateful to Professor P. Pizzo, my thesis advisor, for his valuable suggestions and support throughout the project and also for his encouragement and advice since I was his undergraduate student. I wish to express my appreciation to Dr. F. Sequeda, Dr. H. Notaris, Dr. E. Marinero, Dr. C.J. Lin, Mrs. G. Gorman, and Professor B. Masters for their support and suggestions. 
ABSTRACT

ACKNOFLEDGEMENTS

TABIE OE CONTENTS

IIST OE FIGURES

IIST OF TABIES

CHAPTER 1 INTRODUCTION

1.1. Objective.

1.2. Optical Recording.

1.3. Thin Film Media for Optical Recording.

1.3.1 Phase Change/Microstructure Change.

1.3.2 Magnetization Change.
Page

iii

iv

$\mathbf{v}$

viii

xii

1

1

2

5

5

6

11

15

15

15

16

20

24

26

2.2.2 Signal to Noise Ratio. 
$\begin{array}{ll}\text { Chapter } 3 \text { BACKGROUND. } & 27\end{array}$

3.1. Metallic Multillayers. 27

3.1.1 Crystallographic Orientation of the Layers. 31

3.1.2 Magnetic Properties. $\quad 32$

3.2. Co/Pt Multilayers. 33

Chapter 4 EXPERIMENTAI METHODS. 45

4.1. Experimental Rationale. 45

4.2. Film Deposition and Processing. 45

4.3. Film Characterization. $\quad 47$

4.3.1 Kerr Hysteresis Looper with Heating Stage. 47

4.3.2 Vibrating Sample Magnetometer (VSM). 48

4.3.3 X-Ray Diffractometer. $\quad 50$

Chapter 5 RESULTS AND DISCUSSION. 53

5.1. MO Hysteresis Loop Characteristics. 53

5.2. Structures. 58

5.3. Magnetic Anisotropy. 65

5.4. Kerr Rotation. 68

5.5. Corrosion Resistance. 69

5.6. Effect of Laser Wavelength on Kerr Rotation. 69

5.7. Thermal stability. 72 
Page

Chapter 6 SUMARRY.

Chapter 7 REERRENCES.

75

vii 
Figure 1. Performance Relation Between Optical Disks and other Storage Devices.

Figure 2. Schematic Representation for Comparison of Magnetic and Optical storage Technologies.

Figure 3. The Temperature Occurring in Phase Change Media During Recording, Erasing and Readout. $T_{M}$ and $T_{X}$ are Melting and Crystallization Temperatures, Respectively. C and A Represent Crystalline and Amorphous state.

Figure 4. Transmission Electron Microscope (TEM) Photomicrograph showing Two Written (Amorphous) Domains (in the upper right-hand corner) and Many Erased (crystallized) Domains.

Figure 5. Faraday and Kerr Effects.

Figure 6. Magneto-Optic Recording Process.

Figure 7. Double-sided Magneto-optic Disk

Configuration. Inset Shcis a Lorentz Micrograph of Thermomagnetically Written Domains in RE-TM Film. The Center-to-Center Distance Between the Nearest Domain is $5 \mathrm{\mu m}$.

Figure 8. Three Typical MO Hysteresis Loops.

Figure 9. Schematic Drawing of a Multilayer with Sharp Interfaces. 
Figure 10. Schematic Illustration of the Three

Merwe, b) stranski-krastanov, and c) Volmer-Weber.

Figure 11. Room Temperature saturation Magnetization of Cobalt Platinum Alloys as a Function of Platinum Content.

Figure i2. Coercivity of Cobalt Platinum Alloys as a Function of platinum Content.

Figure 13. Magnetic Hysteresis Loops of Co/Pt Multilayer Films at $300^{\circ} \mathrm{K}$ with Applied Magnetic Field in the Film plane (dashed curve) and Perpendicular to the Film (solid curve).

Figure 14. X-ray Diffraction Patterns for $1 \mathrm{~nm}$ Co and $.25 \mathrm{~nm}$ Pt Layers, $.25 \mathrm{~nm}$ Co and .25 $\mathrm{nm}$ Pt Layers, and .25 $\mathrm{nm} C_{0}$ and $1 \mathrm{~nm}$ Pt Layers (Co $\mathrm{k} \alpha$ radiation).

Figure 15. X-ray Diffraction Ratterns of the Film with $1.5 \mathrm{~nm}$ Co and $1.5 \mathrm{~nm}$ Pt Layers; (a)As-Deposited, (b) After Annealing at $400^{\circ} \mathrm{C}$ for 1 Hour, (c) After Annealing at $600^{\circ} \mathrm{C}$ for 1 Hour. The Peak Observed at $57^{\circ}$ on (c) is Identified as (110) Diffraction of the Co-Pt Alloy.

Figure 16. Schematic Diagram of a MO Kerr Hysteresis Looper.

Figure 17. Schematic Diagram of a Vibrating 51 Sample Magnetometer (VSM).

Figure 18. Low-angle X-ray Diffraction Pattern of the As-deposited Co $(0.3 \mathrm{~nm}) / \mathrm{Pt}(1 \mathrm{~nm})$ Multilayer Film. (Cuka radiation). 
Figure 19. The Effects of Co and Pt Thicknesses on the shape (degree of squareness) of

the Kerr Hysteresis Loop.

Figure 20. Kerr Rotation $\theta_{k}$, Coercivity $\mathrm{H}_{\mathrm{C}^{\prime}}$ and Nucleation Field $\mathrm{H}_{n}$, vs the Thickness of Co Layer in As-deposited Co/Pt Multilayer Films.

Figure 21. Kerr Loop of Co/Pt Multilayers vs Co Thickness.

Figure 22. Kerx Loop of Co/Pt Multilayers vs Pt Thickness.

Figure 23. X-ray Diffraction spectrum of Co/Pt Multilayer Films vs Co Thickness.

Figure 24. X-ray Diffraction spectrum of co/pt Multilayer Films vs pt Thickness.

Figure 25. X-ray Diffraction spectrum of Co/Pt Multilayer Films vs Total Film Thickness.

Figure 26. Standard X-ray Diffraction Spectra of Pt, Co, and CoPt (50:50 at percent) thin films.

Figure 27. Magnetic Hysteresis Loops of Co/Pt Multilayer Films with Applied Magnetic Field in the Film Plane (dashed curve) and Perpendicular to the Film (solid curve).

Figure 28. Kerr Rotation vs Pt Thickness.

Figure 29. Curie Temperature vs Co Iayer Thickness. 
Page

Figure 30. Kerr Rotation vs Reading Wavelength of 73 a co/pt $0.3 / 1.0 \mathrm{~nm} ; 30 \mathrm{~nm} \mathrm{Thick}$ Multilayer Film.

Figure 31. Kerr loop of Co/pt multilayers vs Total Eilm Thickness.

$62 a$ 


\section{IIST OE TABIES}

Table 1. Crystallographic data and differences in atomic distances for a multilayer structure. "Normal" and "Parallel" indicate the directions with respect to the film plane. "-" sign indicates that atomic distance of the co layer is smaller than that of the Pt layer. The crystallographic structure closed with parentheses indicates an expected structure.

Table 2. Constitution, magnetic and Mo properties of Co/Pt multilayer structures. $t_{C o}$ is the co thickness, $t_{\text {Pt }}$ is the Pt thickness, $\theta_{K}$ is the Kerr rotation angle (saturation value), $\mathrm{H}_{c}$ is the coercivity, $\mathrm{Hn}$ is the nucleation field, and $\mathrm{T}_{\mathrm{C}}$ is the Curie temperature. The films are asdeposited. 
Chapter 1

\section{INTRODUCTION}

\subsection{Objective.}

Co/pt multilayer films show promise as high performance, high density erasable magneto-optic recording disks. The primary goal of this thesis is to understand the origin of the unusually large perpendicular magnetic anisotropy of co/pt multilayer thin films and thus to develop film fabrication techniques to improve the magnetooptic and magnetic properties of these films

The thesis is organized as follows: The first three chapters review (1) the principles of optical recording media, (2) properties of multilayer thin films, and (3) prior research on co/pt multilayer films. Chapter 4 presents film fabrication techniques and film characterization methods used in this investigation. Chapter 5 reports properties of the films such as Kerr rotation, magnetic coercivity, magnetization, curie temperature, and corrosion resistance. Finaliy, Chapter 6 contains the summary of results and concluding remarks. 


\subsection{Optical Recording.}

Optical data storage has become a reality in the past few years due to advances in consumer audio and video disk technology and high power semiconductor diode lasers. Optical recording uses laser light to store, erase and retrieve data. It offers high areal bit density, high data rates, random access capability, disk removability, and large head-to-disk separation. High density arises from the ability to focus laser beams to small spots and, at the same time, control the position of the spot accurately. With red or near-infrared lasers and high quality optics, spot diameters of about 1 pm can be obtained, resulting in linear densities on the order of $10^{4}$ bits/cm along the track. A servo-controlled optical system can lock the spot onto a given track with an accuracy of a few tenths of a micrometer providing track densities on the order of $10^{4}$ tracks $/ \mathrm{cm}$. The areal density of the device is thus in the range of $10^{8}$ bits $/ \mathrm{cm}^{2}$, which is more than an order of magnitude better than the current high performance magnetic recording system (1). Since the spacing between the optical head and the disk is as large as $2 \mathrm{~mm}$, in contrast to sub-micron distances in 


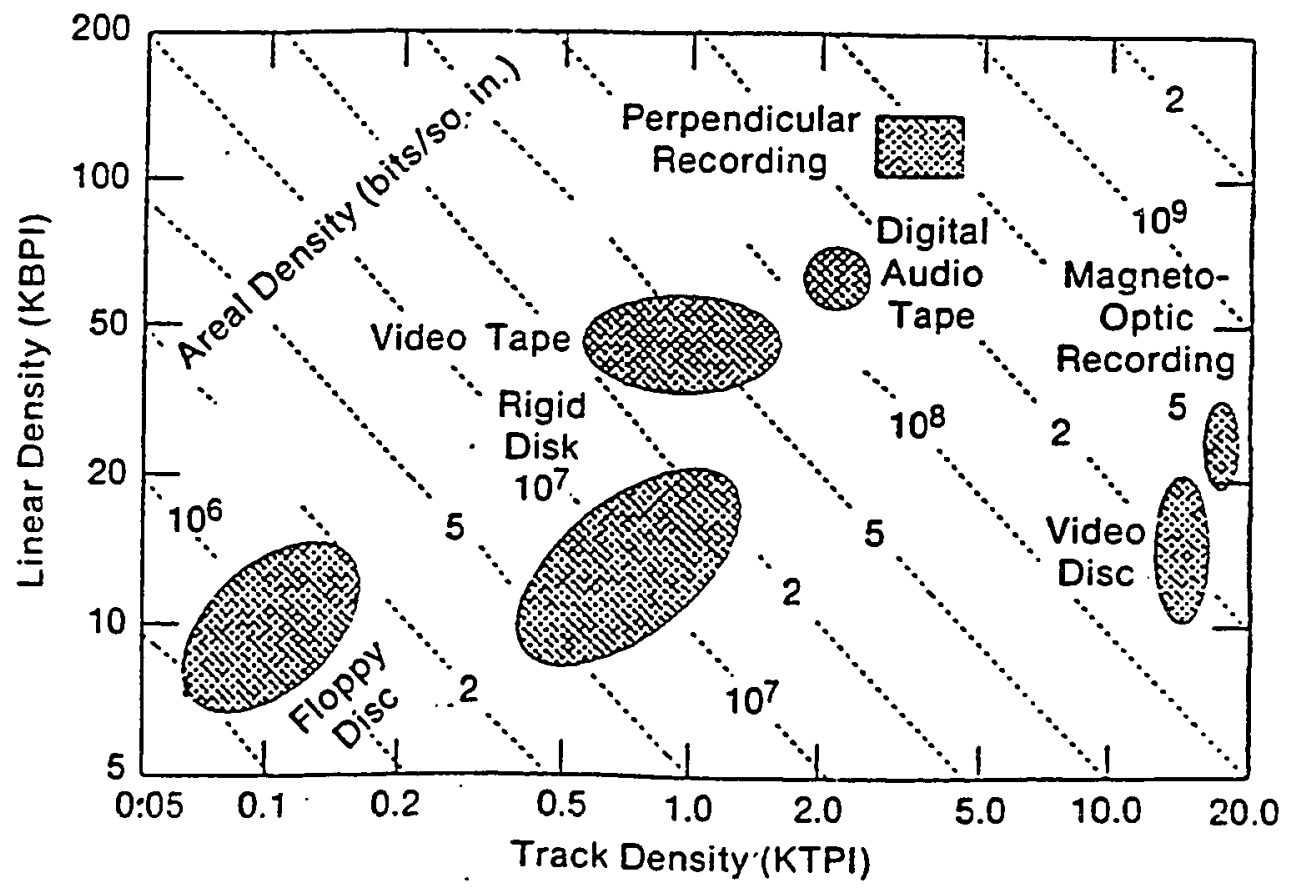

Figure 1. Performance Relation Between Optical Disks and other 


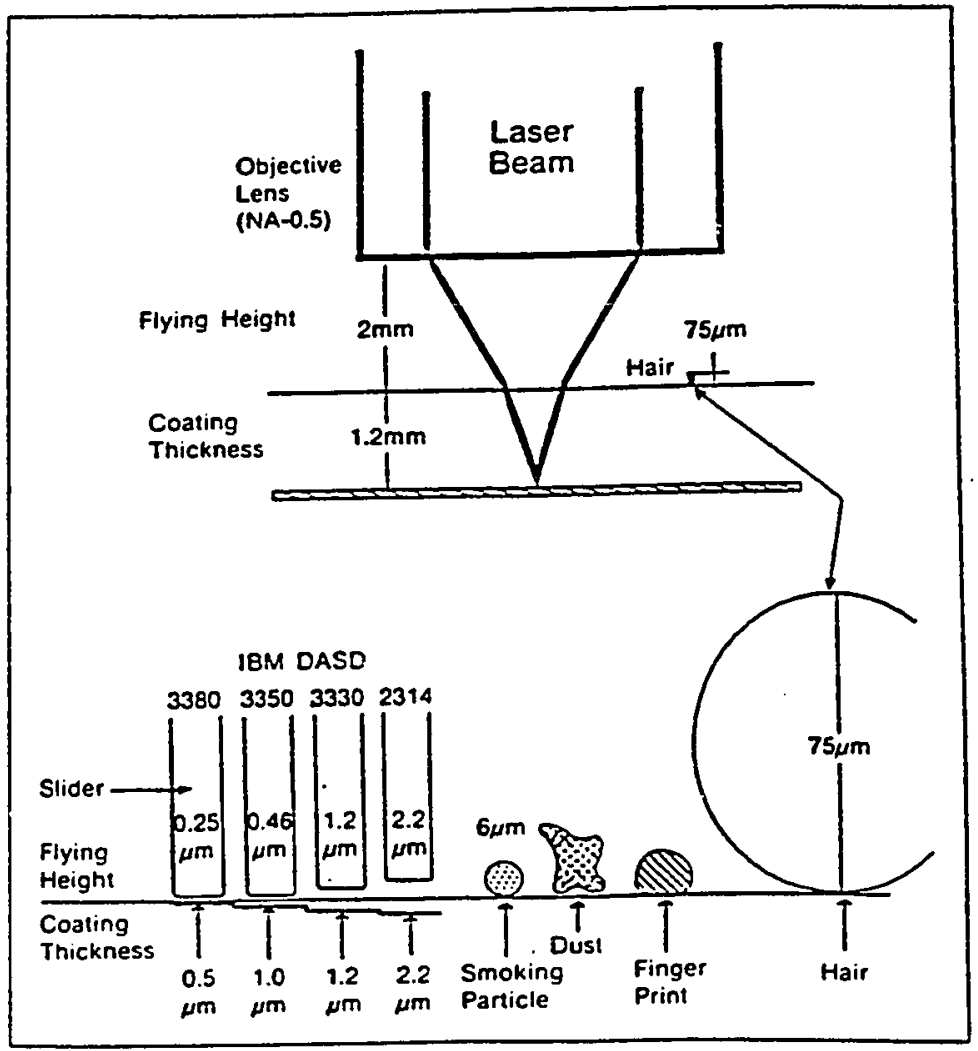

Figure 2. Schematic Representation for Comparison of Magnetic and Optical Storage Technologies. (2) 
magnetic disk drives, the possible head crashes that may occur in magnetic recording drives are eliminated. Laser disk drives are capable of handling high data rates through the use of multiple read/write channels using diode laser arrays. With digital optical storage systems that use readonly and write-once recording media becoming more available, there is increasing interest in reversible media for these systems. Reversible optical data storage has the potential of being used in many applications which are currently served by the traditional magnetic data storage technology.

\subsection{Thin Film Media For Optical Recording.}

The most commonly studied optical recording media can be classified, according to their recording mechanism, into two categories: phase/microstructure change and magnetization change.

1.3.1. Phase Change/Microstructure Change.

This data storage technology is based on the difference of reflectivity between amorphous and crystalline phases of metastable compounds. These materials can be switched 
reversibly between the two distinct structural states by the control of laser power and pulse length. Amorphization (writing) is accomplished by a short ( $<100 \mathrm{~ns}$ ) intense laser pulse. A micron-sized spot is heated to the molten state. At the termination of the pulse, the rate of cooling of this heated spot induced by heat conduction loss is extremely high (about $10^{9}-10^{10} \mathrm{c} / \mathrm{s}$ ). The melted spot is consequently quenched into the amorphous state. The crystallization event (erasure) is achieved by a longer laser pulse of lower power that heats the amorphous material to just below the melting temperature for a long enough time to induce crystallization. A low-power, continuous laser beam is used to observe the change in reflectivity that occurs at the crystalline-amorphous transition (readout). Figure 3 shows the principle of phase change recording. Typical examples of this type of media are InSb-Gasb (3) and $(\mathrm{GeTe})_{x}\left(\mathrm{Sb}_{2} \mathrm{Te}_{3}\right)_{1-\mathrm{x}}$ (4).

1.3.2 Magnetization Change.

Media in this category are the so-called magneto-optic (MO) media. This data storage technology is based either on the Kerr effect (reflection) or the Faraday effect (5) 


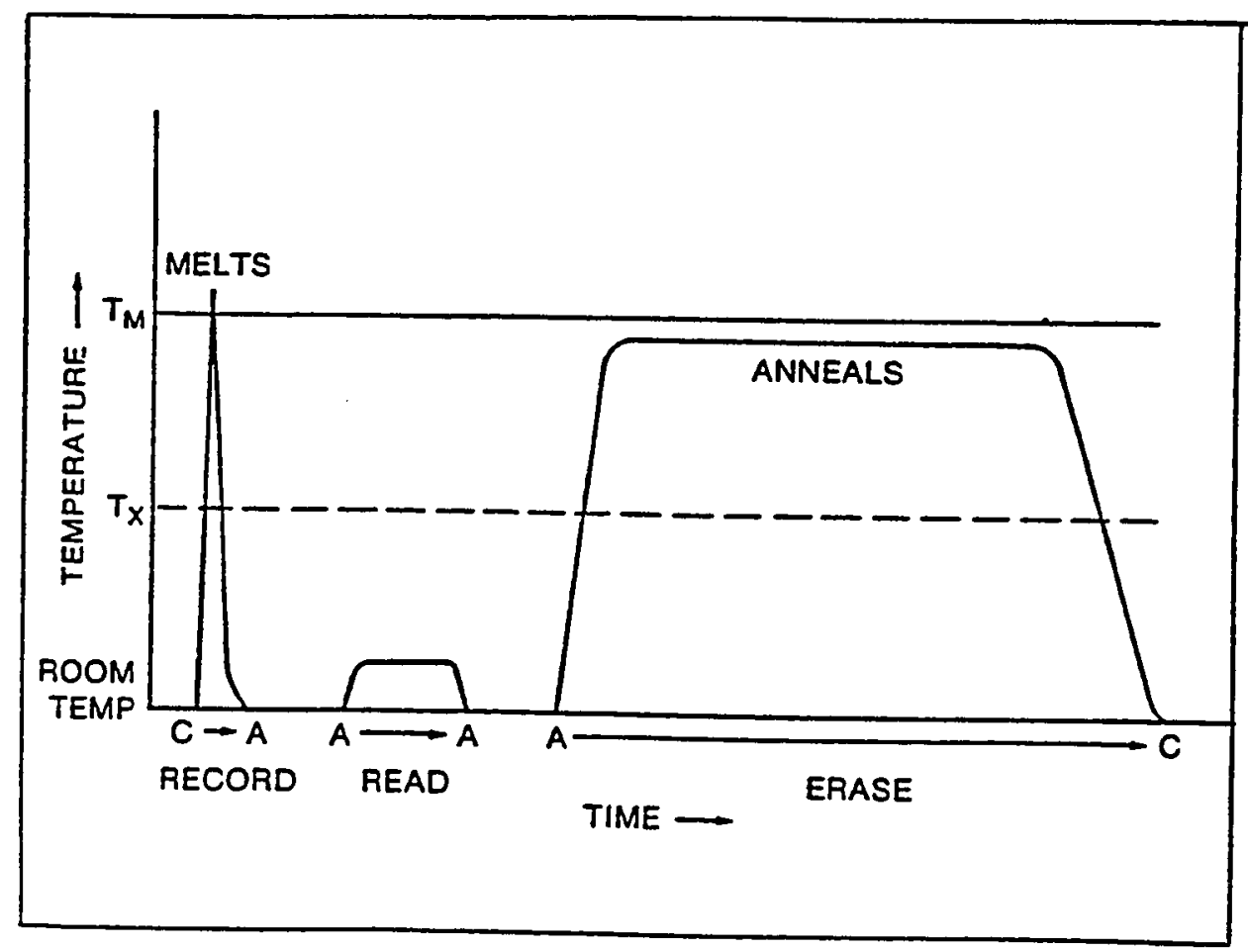

Figure 3. The Temperature Occurring in Phase Change Media During Recording, Exasing and Readout. $T_{M}$ and $T_{X}$ are Melting and Crystallization Temperatures, Respectively. C and A Represent Crystalline and Amorphous State. 


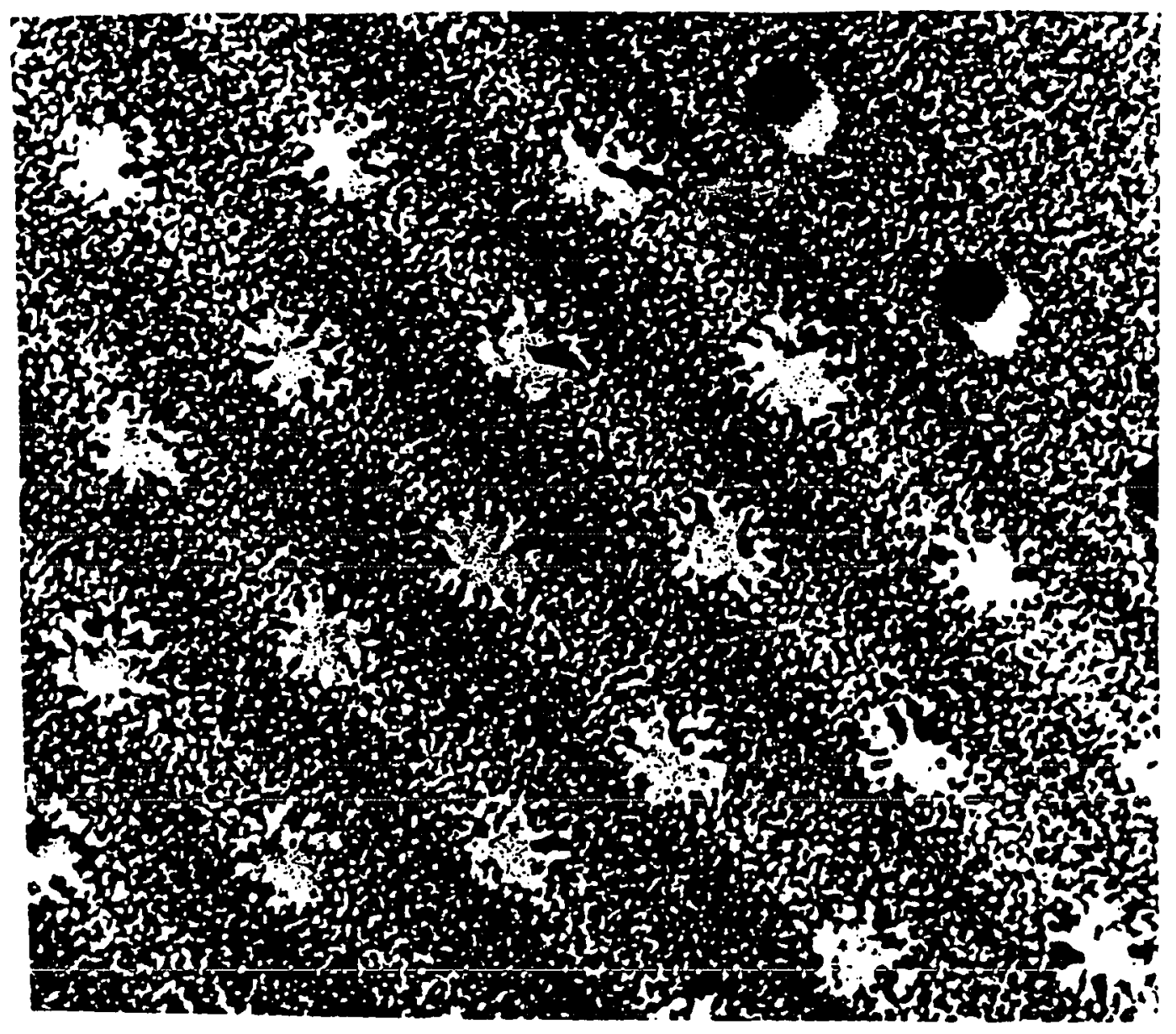

Figure 4. Transmission Electron Microscope (TEM) Photomicrograph Showing Two Written (Amorphous) Domains (in the upper right-hand corner) and Many Erased (crystallized) Domains. 
(transmission). Upon reflection of a linearly polarized laser beam from the material (Kerr effect) or transmission through the material (Faraday effect), the laser beam becomes elliptically polarized with its major axis rotating clockwise or counterclockwise, depending on whether the magnetization component along the beam direction is parallel or anti-parallel to the incident beam direction (see Fig.5). Inside a computer optical disk drive, the reading of information is performed by sensing a linearly polarized laser light of low intensity to sense the change in the magnetization direction of the MO thin film.

Since this magneto-optic recording technology has been chosen for study, it will be discussed in more detail in the following sections. 
. KERR EFFECT (Reflection)

. FARADAY EFFECT (Transmission)

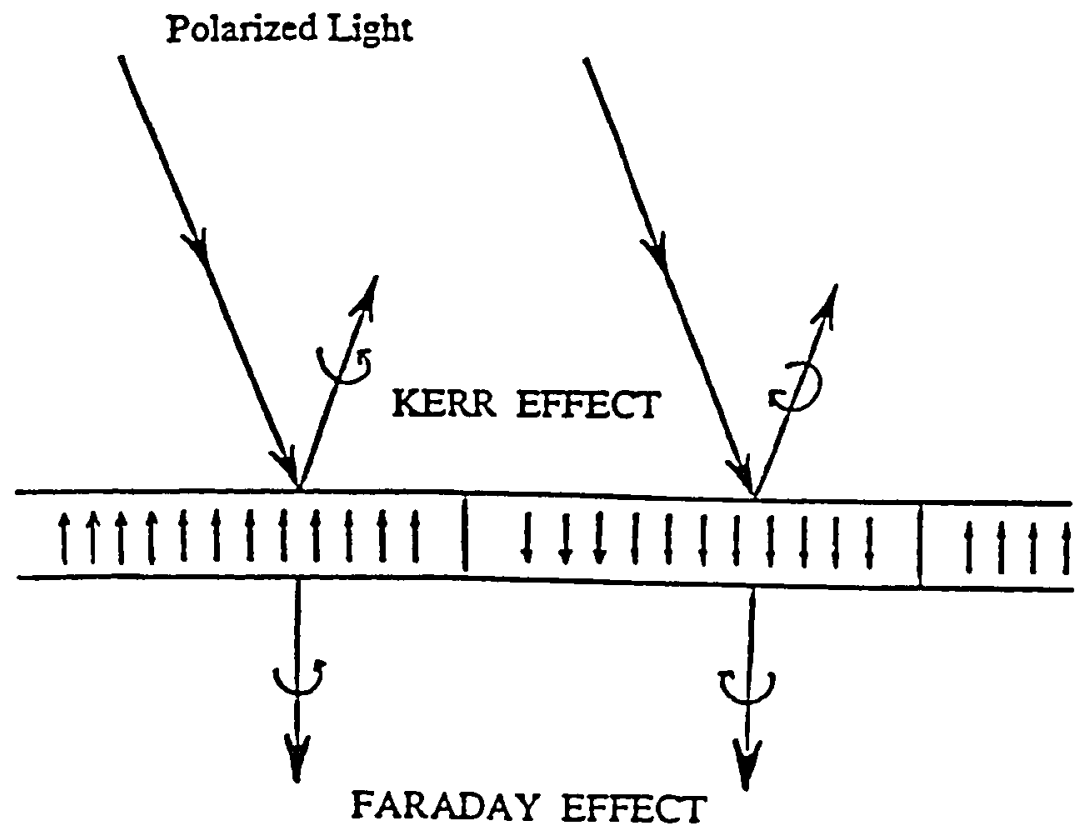

Figure 5. Faraday and Kerr Effects. 
Chapter 2

MAGNETO-OPTIC (MO) RECORDING

In a magneto-optic recording system, recording of information is accomplished by thermomagnetically writing reverse magnetic domains on a pre-saturated medium (all magnetizations are in the same direction). The recording medium normally selected has a magnetic easy axis along the film normal such that the magnetization lies perpendicular to the film plane. The coercivity, which is on the order of thousands of Oersteds at room temperature, decreases with increasing temperature. Using the temperature dependence of coercivity, recording can be achieved by heating a micron or sub-micron spot by focusing a moderately high power (5-10mw) laser beam to a diffraction-limited spot on the medium, under the influence of a small bias magnetic field (on the order of several hundred Oersteds). The bias magnetic field selected is less than the media coercivity at room temperature but greater than the coercivity at high temperatures during laser beam heating. As the local heated spot reaches the switching temperature, with a high enough magnetic field applied antiparallel to the direction of 
magnetization, the magnetization can be reversed locally to form a cylindrical reverse domain. This magnetization reversal does not affect the state of magnetization in neighboring, unheated regions of the film where the coercivity remains high.

The erasure process is very similar to that of writing. By pulsing a focussed laser beam with a power comparable to that used for writing, but with a magnetic field opposite to the direction of the magnetization in the domain, the domain can be made to vanish.

Readout of information employs the magneto-optic Kerr effect(reflection) or Faraday effect(transmission). Iinearly polarized light, reflected(or transmitted) from a perpendicular magnetized medium, is rotated to the left or right, according to the direction of magnetization. The difference in the angle of the plane of polarization for the light reflected (transmitted) is twice the polar kerr rotation angle $\theta_{k}$ (or the Faraday rotation angle $\theta_{F}$ ). The analyzer converts the change in polarization to a change in light intensity, and photodetectors then convert the change into an electrical signal used for readout. Magneto-optic recording processes are shown in Fig. 6 . 


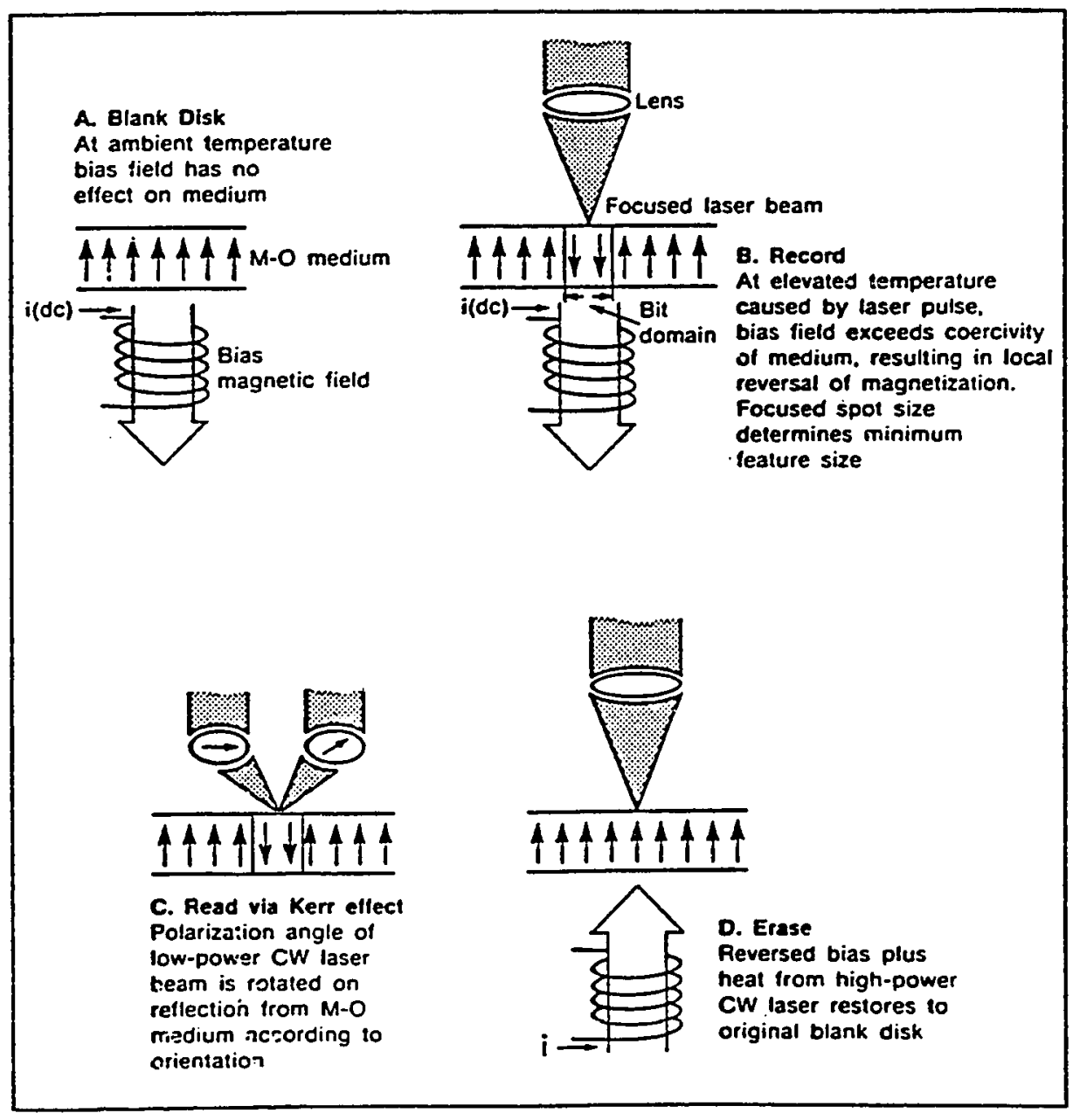

Figure 6. Magneto-Optic Recording Process.

(2) 


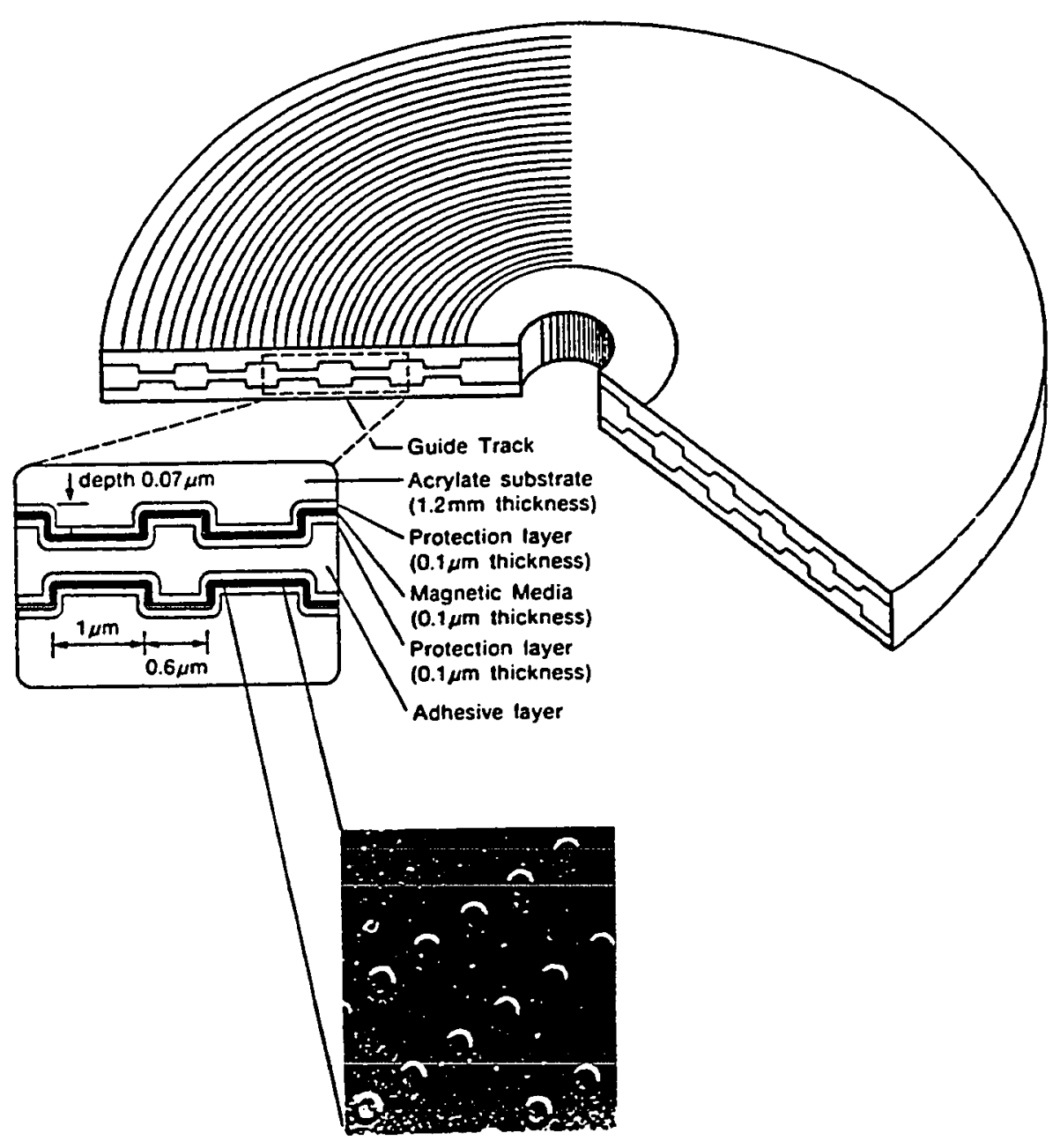

Figure 7. Double-Sided Magneto-Optic Disk Configuration. Inset Shows' a Lorentz Micrograph of Thermomagnetically written Domains in RE-TM Film. The Center-to-Center Distance Between the Nearest Domain is 5 um. (2) 


\subsection{Development of Magneto-Optic Recording.}

2.1.1. Discovery of the Magneto-optic Effect.

In 1845, Michael Faraday discovered that an external magnetic field can rotate the plane of polarization of a plane-polarized light which propagates through a piece of glass. The Faraday effect was the first strong proof of the electromagnetic nature of light. The corresponding effect in reflection was discovered by Kerr in 1888. These effects are all proportional to the net magnetization of the material and not to the external magnetic field strength.

\subsubsection{Early Stages of Magneto-Optic recording.}

The attempts to utilize Mo effects for possible device applications started in the late 50's. At that time, the activities were centered around polycrystalline materials such as MnBi, MnGaGe, and MnAlGe (7). Because of its large Kerr rotation angle, $\left(\theta_{k}=0.7^{\circ}\right) \mathrm{MnBi}$ was one of the early materials that was actively investigated as an Mo recording 
media. Unfortunately, MnBi exists in two crystalographic phases: the $\alpha$-phase at room temperature and the $\beta$-phase at temperature greater than $446^{\circ} \mathrm{C}$. The low temperature phase has a high Curie temperature of $360^{\circ} \mathrm{C}$ which is near the point $\left(446^{\circ} \mathrm{C}\right)$ at which transformation to $\beta$-phase takes place. The high temperature phase has an ideal $10 \mathrm{w}, 180^{\circ} \mathrm{C}$, Curie temperature but it is unstable. It will transform to the more stable a-phase after many writing cycles. Furthermore, despite its large Kerr rotation, the large readback noise associated with the polycrystalline nature of MnBi films leads to a poor signal-to-noise ratio (SNR). The low SNR, poor chemical stability and limited cyclability make MnBi unsuitable for MO recording applications. The fates of Mngage and MnAlGe were similar to that of MnBi because of the excessive read noise cause by random fluctuations in the polarization vector associated with a birefringent micro-crystalline structure.

2.1.3 Recent Developments of Magneto-Optic Recording.

The development of amorphous rare earth-transition metal (RE-TM) thin film alloys (GdCO) in the early 70's by IBM researchers revitalized the future for erasable optical 
recording technology (8). Amorphous magnetic material shows magnetic ordering, but no evidence of long-range atomic ordering. The magnetic ordering of GdCo alloys is an antiferromagnetic exchange coupling between RE(Gd) and TM(Co) sub-networks. The absence of crystalline structure in amorphous materials results in an improved SNR because the media noise is much lower than that of polycrystalline films. But amorphous GdCo later proved to be unsuitable for high density Mo recording applications because its coercivity was not high enough over a wide enough temperature range, to support domains with diameter less than $2 \mu \mathrm{m}$ (9).

The invention and successful development of high power reliable diode lasers coupled with the rapid progress in non-erasable optical recording in the early 80 's attracted further and extensive research and development efforts in MO recording technology.

The search for new Mo materials has been focusing on different binary, ternary and quaternary alloy systems derived from the earlier binary RE-TM alloy (8). Steady progress in the optimization of composition of the RE(Tb, Gd, DY)-TM(Fe, Co) alloy films made them the most promising and highly developed media for magneto-optic data storage 
(10-14). The as-deposited RE-TM films are amorphous over a wide range of RE/TM ratio. The perpendicular magnetic anisotropy of the films can be optimized by varying the RT/TM ratio. And the Curie temperature is controlled by the Co/Fe ratio. The absence of crystalline grains and grain boundaries of RE-TM amorphous films eliminates readback noise and leads to a high SNR even though the Kerr rotation of these films is less than 0.3 degree.

However, the RE-TM alloys have several deficiencies. The most notable is the easy oxidation of the rare earth metal components with consequent loss of the magnetic properties required in recording. Protective layers only further complicate the structure and encumber manufacture. Another limitation, if higher storage densities are to be achieved using shorter-wavelength lasers, is the decrease of the Kerr rotation of RE-TM alloys at shorter wavelengths. These drawbacks have led researchers to continue to look for other materials.

Among all known materials with the potential of offering large Kerr of Faraday rotation, polycrystalline Bisubstituted garnets $\left[(\mathrm{BIR})_{3}(\mathrm{FeM})_{5} \mathrm{O}_{12}\right](\mathrm{R}=\mathrm{Y}, \mathrm{DY} ; \mathrm{M}=\mathrm{Ga}, \mathrm{Gd})$ could potentially replace or complement the RE-TM alloys. Just like RE-TM alloys, the Curie temperature and perpendicular 
magnetic anisotropy of the garnet films are affected by chemical variation (15). However, the chemical stability of garnet films offers advantage over the RE-TM alloys. Also, the garnets offer a large Faraday rotation at short wavelength which will be needed for future high density recording. The major drawback of the polycrystalline garnet is its large readback noise arising from the orientation variation of constituent grains and grain boundaries, and the related domain irregularity. Most research efforts so far have been directed at reducing the size of crystal grains by either high temperature deposition or postdeposition anneal (16-17).

Heusler alloys, especially PtMnSb as recently reported by Engen and Co-workers (18), exhibit a large Mo Kerr rotation $\left(\theta_{k}=2^{\circ}\right)$ at room temperature. They are environmentally durable and are thus attracting interest for use as magneto-optical recording media. Pt, $\mathrm{Mn}$, and $\mathrm{Sb}$ are non-magnetic, but the PtMnSb compound is ferromagnetic. zener (19) assumed that the origin of ferromagnetism of this alloy is from the indirect coupling of incomplete d-shelis on Mn atoms via the conducting electrons and, or atomic orbitals of Pt and Sb atoms. Despite the large $\theta_{k}$, PtMnSb films still are not used as a Magneto-Optic recording media 
because of their low perpendicular magnetic anisotropy. Various research and development efforts have been applied to improve the magnetic properties of the film. So far, highly perpendicular anisotropic PtMnSb thin films have not been obtained.

Metallic multi-layered films such as Co/Pt, Co/Pd, and Co/Au $(20-21$ ) are also quite promising for Mo recording applications. Multilayer films with alternating layers of Co, and a non-magnetic noble metal, each a few nanometers thick, have been shown to possess high perpendicular anisotropy. These films are attracting attention because of the improved Kerr rotation at short wavelength and the high environmental stability over the RE-TM alloy films. Since the Colpt multilayer system has been chosen for study in this thesis, research on this material carried out by other investigators will be discussed in detail in Chapter 3 .

\subsection{Requirements for Maqneto-Optic Thin Film}

Magnetic coercivity, $\mathrm{H}_{C^{\prime}}$ is an important property of a Mo thin film. Any film used as a Mo recording media must have high $\mathrm{H}_{\mathrm{C}}$ at room temperature to support micron or submicron sized magnetic domains for high areal density 
storage. The value of $\mathrm{H}_{\mathrm{C}}$ is calculated from the magnetic or Kerr hysteresis loops obtained in the vibrating sample Magnetometer (VSM), or the Kerr looper, a device which measure the Kerr rotation angle and the coercivity of the Mo film. Typical Kerr hysteresis loops are shown in Fig. 8.

The shape of these loops is also important to Mo recording. A non-square loop characterizes a medium with high background noise and this leads to a low signal-tonoise ratio (SNR). The shape of the hysteresis loop is characterized by three fields: the coercivity field $\mathrm{H}_{\mathrm{C}^{\prime}}$ the saturation field $\mathrm{H}_{S}$ and the nucleation field $\mathrm{H}_{n}$, terms indentified in Fig. 8. The nucleation field $H_{n}$ is the field where nucleation of a reversed domain starts. It is here defined as positive if this occurs at an applied field whose sign is opposite to that of the magnetization direction. That is if $\mathrm{H}_{\mathrm{n}}>0$, the remanence is 1008; if $\mathrm{H}_{\mathrm{n}}$ is small or negative, the domain structure of the film is not stable. The saturation field $\mathrm{H}_{S}$ is the field where all the domains are saturated and have the same direction. If $H_{S}$ is large than $\mathrm{H}_{C^{\prime}}$ a higher field than $\mathrm{H}_{C}$ is required to saturate the domains. This also contributes to the media noise.

A good magneto-optic recording medium should have (1) a high enough coercivity to support micron or even sub-micron 

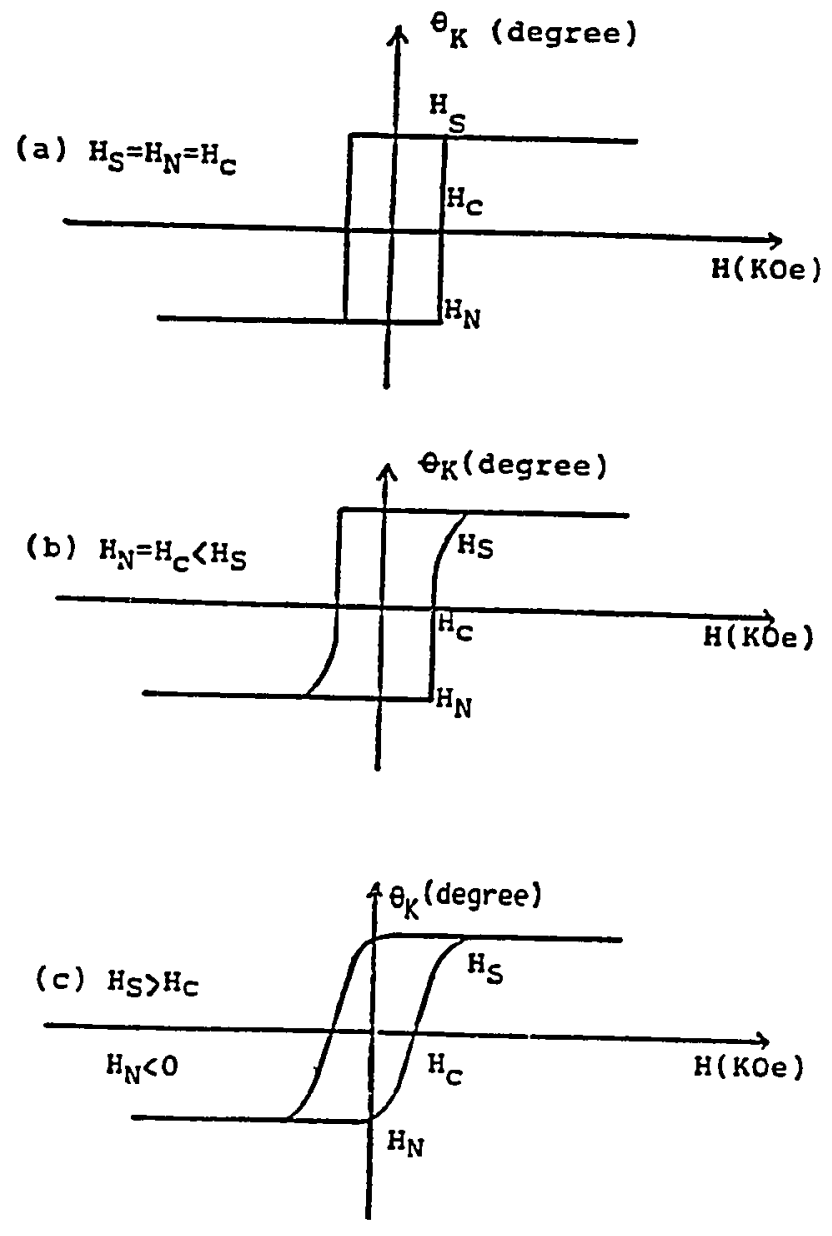

Figure 8. Three Typical MO Hysteresis Loops. 
sized magnetic domains for high areal density storage, (2) a good recording performance, i.e., high signal-to-noise ratio (SNR) to support a high bit density and high data rates, (3) good writing sensitivity, which is important for achieving high data rates when semiconductor lasers are used, and (4) good long-term structural and environmental stability. Also, the media used. should exhibit a uniaxial anisotropy with the preferred axis of magnetization perpendicular to the plane of the film, together with appropriate temperature dependence of coercivity and magnetization as well as a large coercivity at room temperature. It must also have a sufficiently large light absorption coeffecient at the wavelength of the write laser whereby the thermomagnetic effect can be utilized for writing and erasing. With these constraints, the general requirements for a suitable Mo material are as follows: (23)

- A large magneto-optic effect, but one which is not strongly temperature dependent.

- A sufficiently large perpendicular uniaxial anisotropy (designated as $\mathrm{Ku}$ ) such that the preferred orientation of magnetization is perpendicular to the plane of the film. 
- A large coercivity (HC) at room temperature, but which reduces to a low enough value at high temperature to allow magnetization switching.

- A light absorption coefficient sufficient to provide a convenient write/erase energy threshold, but not so high as to restrict readout of the reflected beam.

- Reusability (more than $10^{6}$ write/erase cycles) and a minimum of 10-year lifetime

- Manufacturability, good homogeneity, scale-up capability, and

. Low cost.

2.2.1 Bit Density.

Several factors determine the bit density in mo recording (23). They are (1) the properties of the recording medium (anisotropy energy, saturation magnetization, and coercivity), (2) the wavelength of the laser, and (3) the numerical aperture of the objective lens. The smallest stable domain size at zero applied field sets the ultimate bit density.

The minimum stable domain diameter $d_{0}$ at zero applied field is determined in part by the domain wall energy, $\sigma$ 


$$
\sigma=4 \mathrm{~A} \sqrt{\mathrm{K}_{\mathrm{v}}}
$$

where A: constant

$\mathrm{K}_{\mathrm{u}}$ : Uniaxial perpendicular anisotropy energy constant.

$\mathrm{Ku}$ is related to the orientation of the magnetization and the structure of the film. It is measured by a torque magnetometer.

The value of $d_{0}$ is given by:

$$
\mathrm{d}_{0}=\sigma / \mathrm{M}_{\mathbf{s}} \mathrm{H}_{\mathrm{C}}
$$

where $M_{s}$ : Saturation Magnetization

$\mathrm{H}_{C}$ : Coercivity

The size of the laser spot must be smaller or equal to the domain size. The laser spot size is given by:

$$
\mathrm{d}=0.55 \lambda / \mathrm{NA}
$$

where $\lambda$ : laser wavelength

NA: numerical aperture of the objective lens

Decreasing the wavelength, $\lambda$ and increasing the numerical aperture will decrease the laser spot size. For example, for a diode laser of wavelength $820 \mathrm{~nm}$ and an objective lens with $\mathrm{NA}=0.6$, the laser spot sizt is about 
$0.75 \mathrm{\mu m}$. This leads to a maximum areal bit density of about $1 \times 10^{8}$ bits $/ \mathrm{cm}^{2}$.

2.2.2 Signal to Noise Ratio (SNR)

To maximize the SNR, the signal must be maximized and the noise minimized. The noise is caused by media, laser and electronic contributions. Media writing noise results from domain irregularity; ie, the non-square shape of the magnetic hysteresis loop, for example.

The readout signal is proportional to $R \theta_{k}{ }^{2}$ (23). The signal increases when the kerr rotation, $\theta_{k}$, and the reflectivity, $R$, of the Mo film increase. To obtain a high signal, the $\theta_{k}$ and $R$ values of the film must be as high as possible.

Rare earth-transition metal amorphous thin films which are being used in commercial optical disk drive today have low Kerr rotation $\left(\theta_{k}<0.3^{\circ}\right)$; but the CNR* of these amorphous films is high (60dB) (24) because of the low media noise due to their amorphous structure and their high value of reflectivity, $R$.

* CNR: Carier-to-noise ratio, one part of SNR, is frequently used to determine recording performance of a Mo disk. 
Chapter 3

BACKGROUND

\subsection{Metallic Multilayer systems.}

Metallic multilayer films are thin films in which the composition is modulated by alternating deposition of different metallic materials. The films are usually prepared by conventional vacuum deposition methods such as evaporation and sputtering. The alternation between two materials can be done gradually to obtain a smooth transition of one material into the other or abruptly to obtain a sharp interface.

Fig. 9 shows a schematic representation of a multilayer system. The multilayer system can be characterized by the individual layer thicknesses, $t_{A}$ and $t_{B}$, and the total number of bilayers(N). A typical feature of the multilayer medium is that in the direction perpendicular to the film plane the structure repeats itself with a certain period, called the modulation length $D$, which, in this case, is equal to the sum of the layer thicknesses $t_{A}+t_{B}$. The individual thicknesses range from approximately 0.1 to $10 \mathrm{~nm}$ 


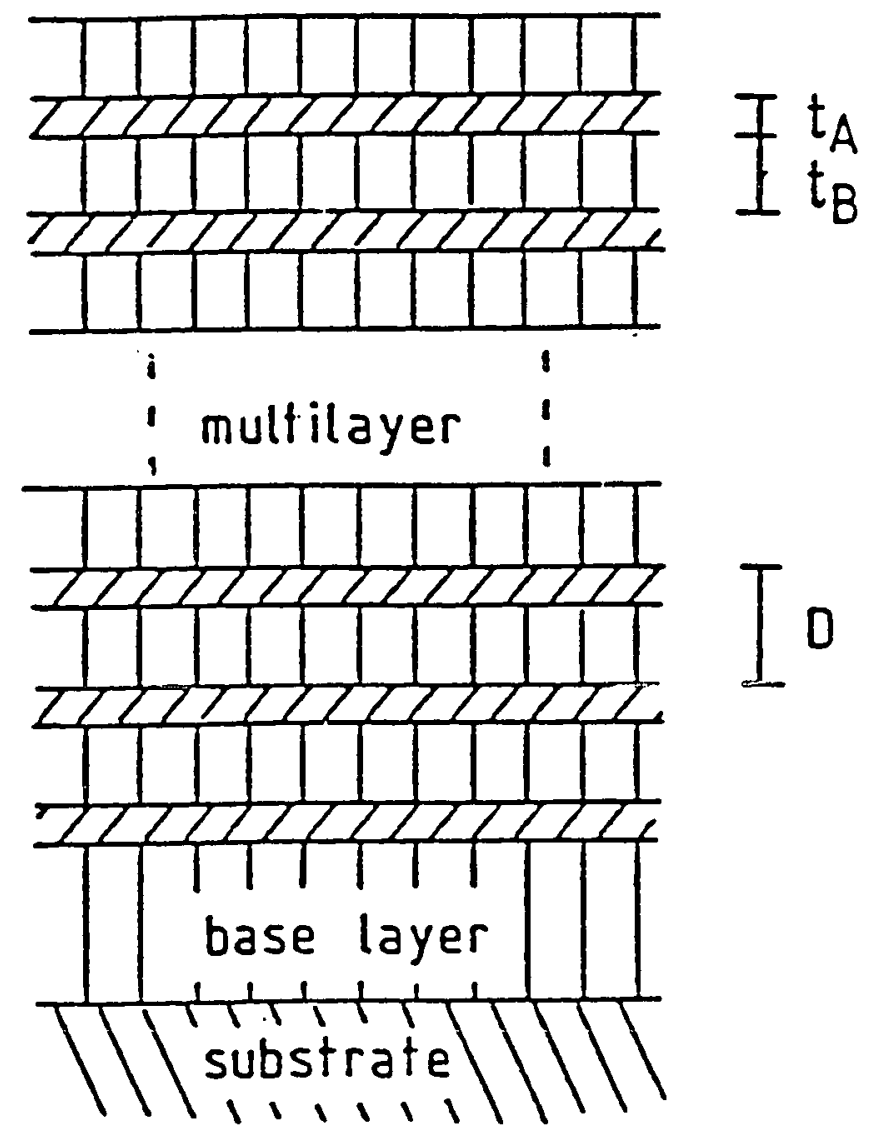

Figure 9. Schematic Drawing of a Multilayer with Sharp Interfaces. 
which means from less than one to several atomic layers. The total thickness of the film is typically about $30 \mathrm{~nm}$.

The individual layers can be amorphous or crystalline, and they can have the same or different crystal structure. Depending on the deposition rate, the temperature and other deposition parameters, the individual layers grow differently. On a microscopic scale, there are usually four steps in the growth of a thin film: 1) nucleation, 2) outgrow of the nuclei into islands, 3) coalescence of islands, and 4) filling of channels (25). The way in which these stages are completed depends on the growth mechanism involved. Three major growth mechamism have been distinguished and they are illustrated in Fig. 10 (26): (1) The Frank-Van der Merwe (FvdM) mechanism, (2) The Volmer-Weber(VW) mechanism, and (3) The Stranski-Krastanov(SK) mechanism. In the Frankvan der Merwe mechanism, atomic layers grow layer-by-layer; that is, the four stages are completed before a new atomic layer nucleates. In the Volmer-Weber mechanism islands grow without complete and uniform coverage of the substrate. In the Stranski-Krastanov mechanism initially one or more atomic layers are formed; but then islands grow without covering the full film. The occurrence of the appropriate growth mechanisms of Fig. 10 depends on the materials 


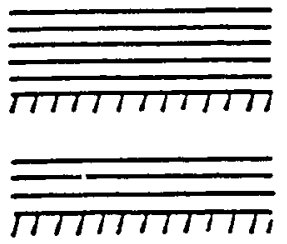

$\overline{\text { ताग }}$

तTाTाTाभ
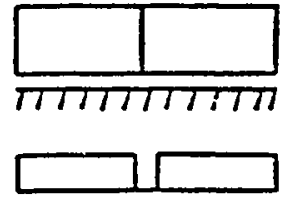

TाTाITाT,

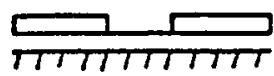

TाтTाтT.
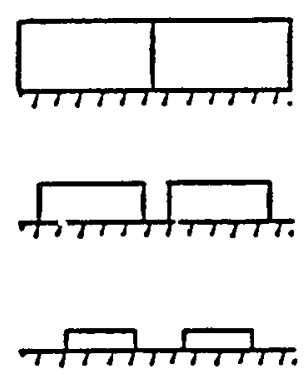

(c)

(a)

(b)

Figure 10. Schematic Illustration of the Three Growth Mechanisms: a) Frank-Van der Merwe, b) Stranski-Krastanov, and c) volmer-Weber. 
involved, the temperature and crystallographic orientation of the substrate, the deposition rate, the kinetic energy of the incoming particles, the bombardment of the substrate by other particles, and other factors. To make a multilayer of materials $A$ and $B$ for Mo application the FvdM mechanism for the growth of $A$ on $B$ and for $B$ on $A$ is usually preferred.

\subsubsection{Crystallographic Orientation of the Layers.}

The orientation of the crystallites in the layer relative to the film plane can by 1) epitaxial, 2) textured or 3) random. The deposition of multilayers starts with a base layer on the substrate which has either an amorphous or crystalline surface structure. When a material is deposited onto a crystalline substrate or base layer, two types of epitaxial growth may occur: 1) strained layer growth or 2) pseudomorphic growth.

1) Strained layer growth occurs when there is a small lattice mismatch between the similar crystal structures of the different materials. An expansion in the plane of the layer is accompanied by a contraction perpendicular to it and vice versa. This type is theoretically predicted by van der Merwe up to a certain layer thickness of a few atomic 
layers (27). Above this critical thickness ( $1 \mathrm{~nm}$ ) dislocations will relax the strain.

2) In pseudomorphic growth, the crystal structure of the material at the surface is transferred to that of the deposited under-layer. This is the case for Fe deposited on $\mathrm{Cu}(111)$ and on $\mathrm{Cu}(100)$. Fe deposited on copper does not adopt the bcc structure, but the fcc structure up to a certain thickness (28)

\subsubsection{Magnetic properties.}

The magnetic properties of multilayers in which one of the layers is ferromagnetic in its bulk form are influenced by several effects which do not exist in the bulk magnetic material. First of all, there are interfacial effects, that is, effects depending on the kind of material that is neighboring the magnetic layer. These interfaces can reduce or enhance the net magnetic moment of the layers. A more important effect of the interface is that the anisotropic environment can change the preferred orientation of the magnetization. If the magnetic layers are sandwiched between non-magnetic layers, long range magnetic coupling 
across the non-magnetic layers may occur, and this leads to interesting new spin structures (29).

Finally, structural modifications, induced by neighboring layers might create metastable phase which have a different crystal structure and may thus lead to new magnetic properties.

\subsection{Co/Pt Multilayers Thin Films.}

Copt alloys have been used as permanent magnets because of their high magnetic coercivity and magnetization. Numerous reports on Copt alloy thin films and their respective magnetic properties and crystal structures have been reported (30-31). But CoPt alloy was not an attractive candidate for Mo recording media because of low perpendicular magnetic anisotropy and non-squareness of the magnetic hysteresis loop. However, in the past couple of years, there have been increasing research efforts on cocontaining multilayer films such as Co/Pt, Co/Pd, and Co/Au (21-22). This is because high perpendicular magnetic anisotropy has been achieved in these multilayers. The nature of the high perpendicular anisotropy in these multilayer structures has not been fully understood. 
According to D. Treves et al (30), Copt alloy thin films prepared by $R F$ Sputtering at.room temperature exibit fcc crystal structure, in-plane magnetization, and low coercivity $\left(\mathrm{H}_{C}<1 \mathrm{KOe}\right)$. This is in contradiction to the bulk properties of CoPt: bulk CoPt near the 50/50 at percent composition is tetragonal below $833^{\circ} \mathrm{C}$ and fcc above. Films deposited at high substrate temperature $\left(500^{\circ} \mathrm{C}\right)$ or deposited at room temperature and post-annealed at $600^{\circ} \mathrm{C}$ have mostly tetragonal structure and do show perpendicular magnetization. The coercivity of these films is high (up to 8Koe); but the shape of their magnetic hysteresis loops is not square. The ratio of remanence to saturation magnetization, a measure of the "squareness" of the hysteresis loop, is about 0.8 .

J.A. Aboaf et al. [31] studied the properties of RF sputtered Copt alloy thin films as a function of Pt content from 0 to 80 atomic percent. In the range $0-32$ and $40-80$ at. percent $\mathrm{Pt}$, the films are ferromagnetic. However, for Pt contents between 32 to 40 at. percent, the films are nonmagnetic as is shown in Fig. 11 and 12. Aboaf et al. assume that the discontinuity in the magnetic behavior is the result of a Curie temperature which is below room temperature for Pt content of 32-40 atomic percent rather 


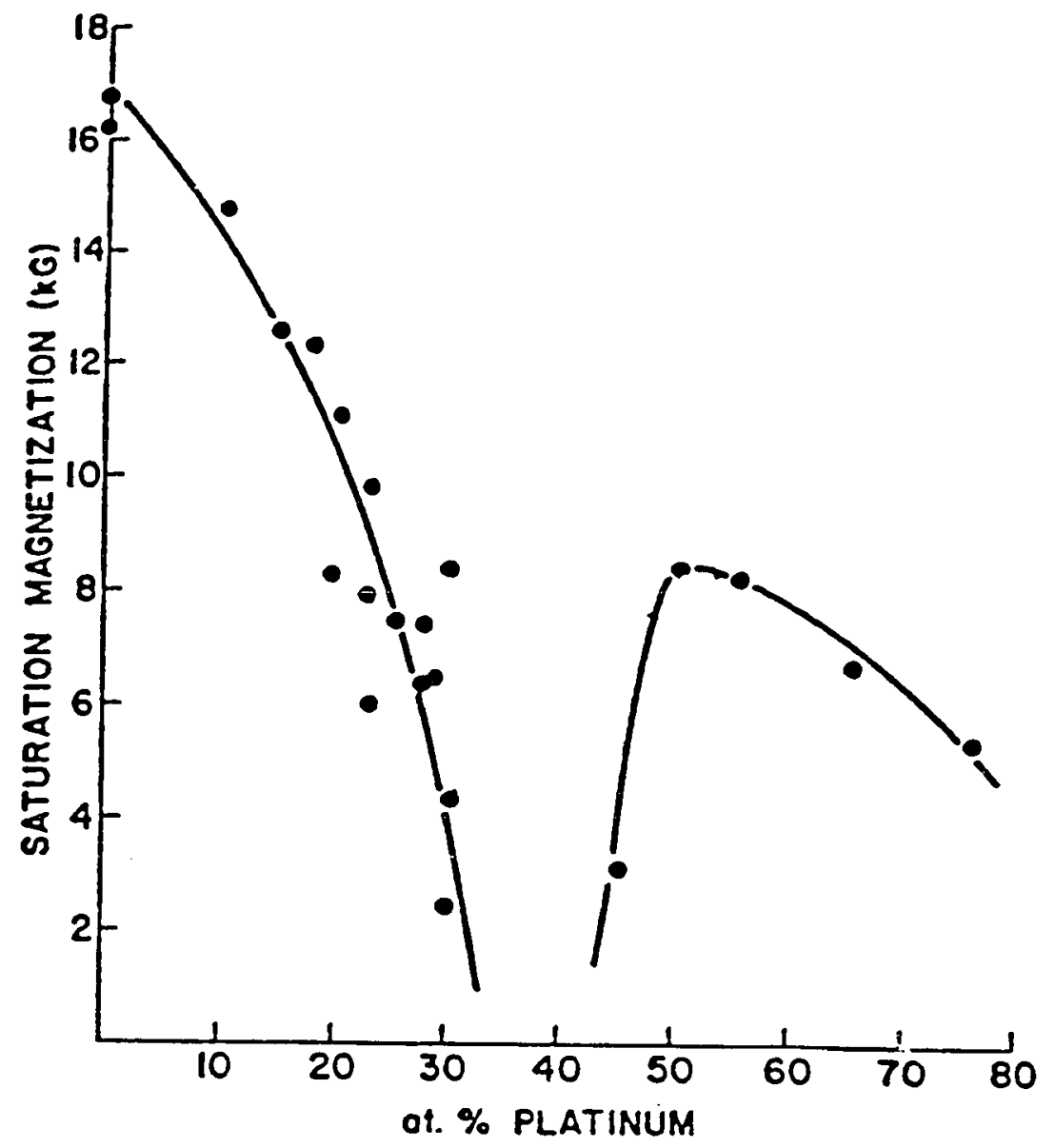

Figure 11. Room Temperature Saturation Magnetization of Cobalt Platinum Alloys as a Function of Platinum Content. (31) 


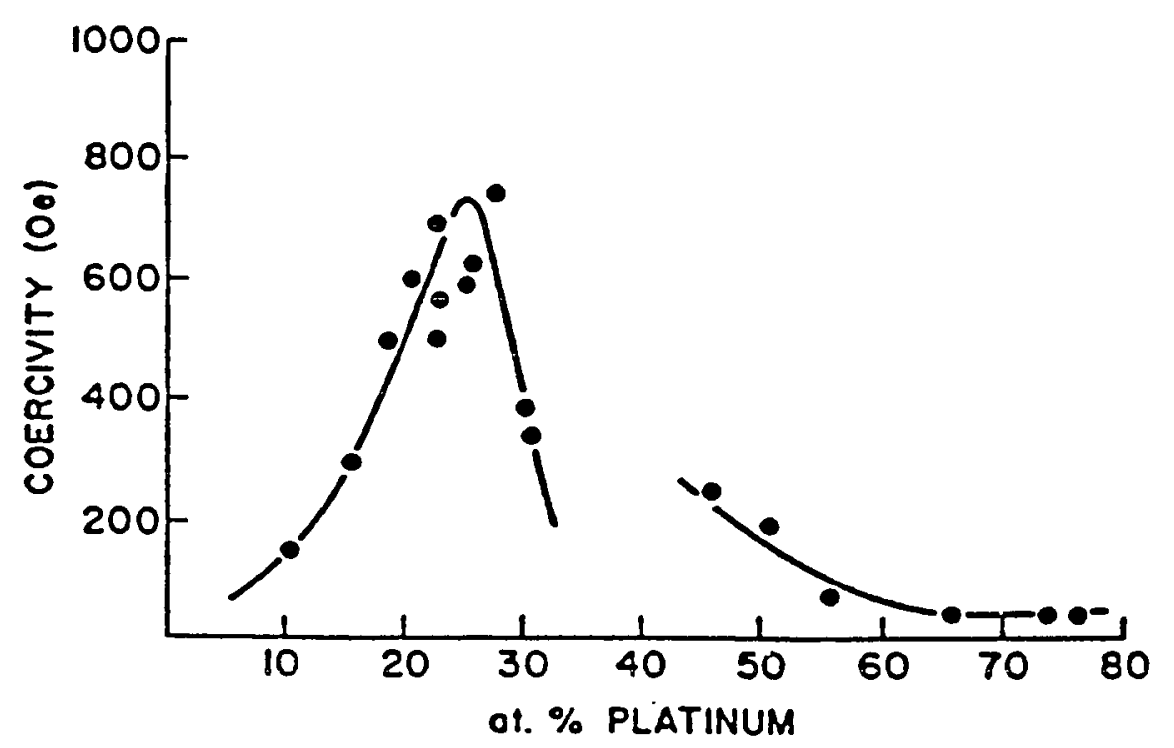

Figure 12. Coercivity of Cobalt Platinum Alloys as a Function of Platinum Content. (31) 
than a reduction in the cobalt magnetic moment by platinum addition. Structural data for these non-magnetic Copt films were not reported.

The structure of CoPt films containing about 25 at percent Pt is a mixture of hexagonal and face-centered cubic (FCC) phase. The coercivity of these films is large (700 to 2000 Oe) and does not change with heat treatment up to a temperature of $600^{\circ} \mathrm{C}$; but the coercivity decreases rapidly at $700^{\circ} \mathrm{C}$. The properties of 50:50 CoPt alloy thin films are similar to those of bulk alloys and their coercivity increase upon heat treatment. These films contain an ordered tetragonal phase in the face-centered cubic matrix. The films which contain about 75 at percent $P t$ have a disordered fcc phase and with heat treatment at $500^{\circ} \mathrm{C}$ an ordered fcc phase forms. The coercivity of these film is small (200 oe) and does not change upon annealing.

Research on multilayer thin films containing co and noble metals (Pd, Au) began about 1985 (20-21); but work on the CoPt multilayer structures is very recent.

P.F. Carcia (20) states that sputtered Co/Pt multilayer films are polycrystalline and highly textured with a (111) orientation along the growth direction. The films exibit perpendicular magnetic anisotropy when the co layer is 
thinner than $1.4 \mathrm{~nm}$; and the magnetization of the films is higher than the bulk Co. The coercivity of these films is small, less than $0.5 \mathrm{kOe}$. And the remanent magnetization is much smaller than the saturation magnetization; that is, these films exhibited a low degree of squareness (see Fig. 13). After annealing in vacuum at $400^{\circ} \mathrm{C}$ for 2 hours, the in-plane direction becomes the easy axis of magnetization and the magnetization increases $15 \%$.

N. Sato (32) studied the crystallographic structure and magnetic properties of Co/Pt multilayers. These films were made by the sputtering technique. Films with a small periodicity have either a hop structure with the c-axis normal to the film plane or a fcc structure with the (111) axis normal to the film plane, depending on the thickness ratio of Co to $\mathrm{Pt}$ (see Table 1). The structure of $\mathrm{Pt}$ in a film with a thicker co layer than the Pt layer changes from fcc to hcp and the structure of co in films with a thinner Co layer than a Pt layer changes from hop to fcc [See Fig. 14]. Fig. 15 shows the change in crystalline diffraction of the film with $1.5 \mathrm{~nm} C o$ and $1.5 \mathrm{~nm}$ Pt layers. The $\mathrm{x}-\mathrm{ray}$ diffraction pattern of the as-deposited film contains one main peak [fcc(111)CoPt]. Two satellite peaks, one that correspond to the (111) diffraction of fcc-pt and the other 


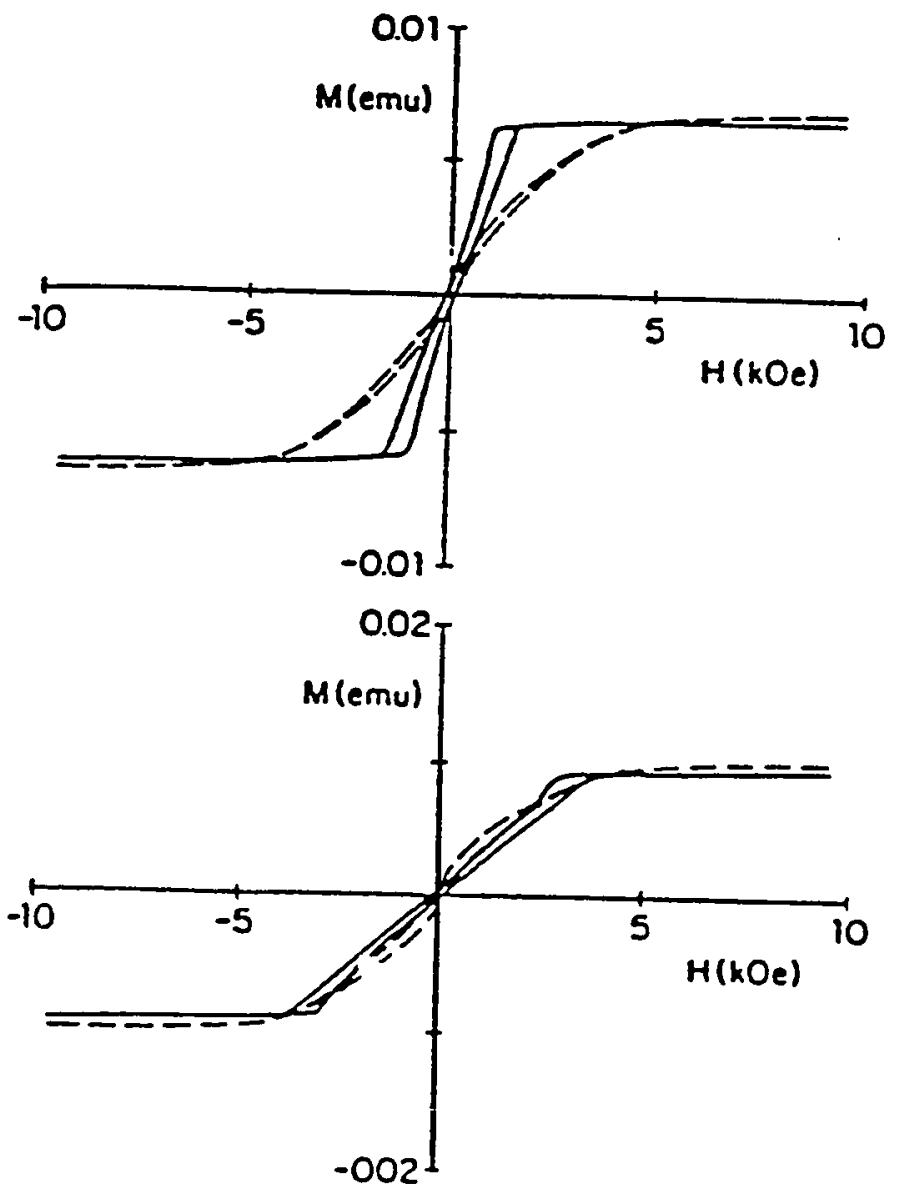

Figure 13. Magnetic Hysteresis Loops of Co/Pt Multilayer Films at $300^{\circ} \mathrm{K}$ with Applied Magnetic Field in the Film Plane (dashed curve) and Perpendicular to the Film (solid curve). (20) 


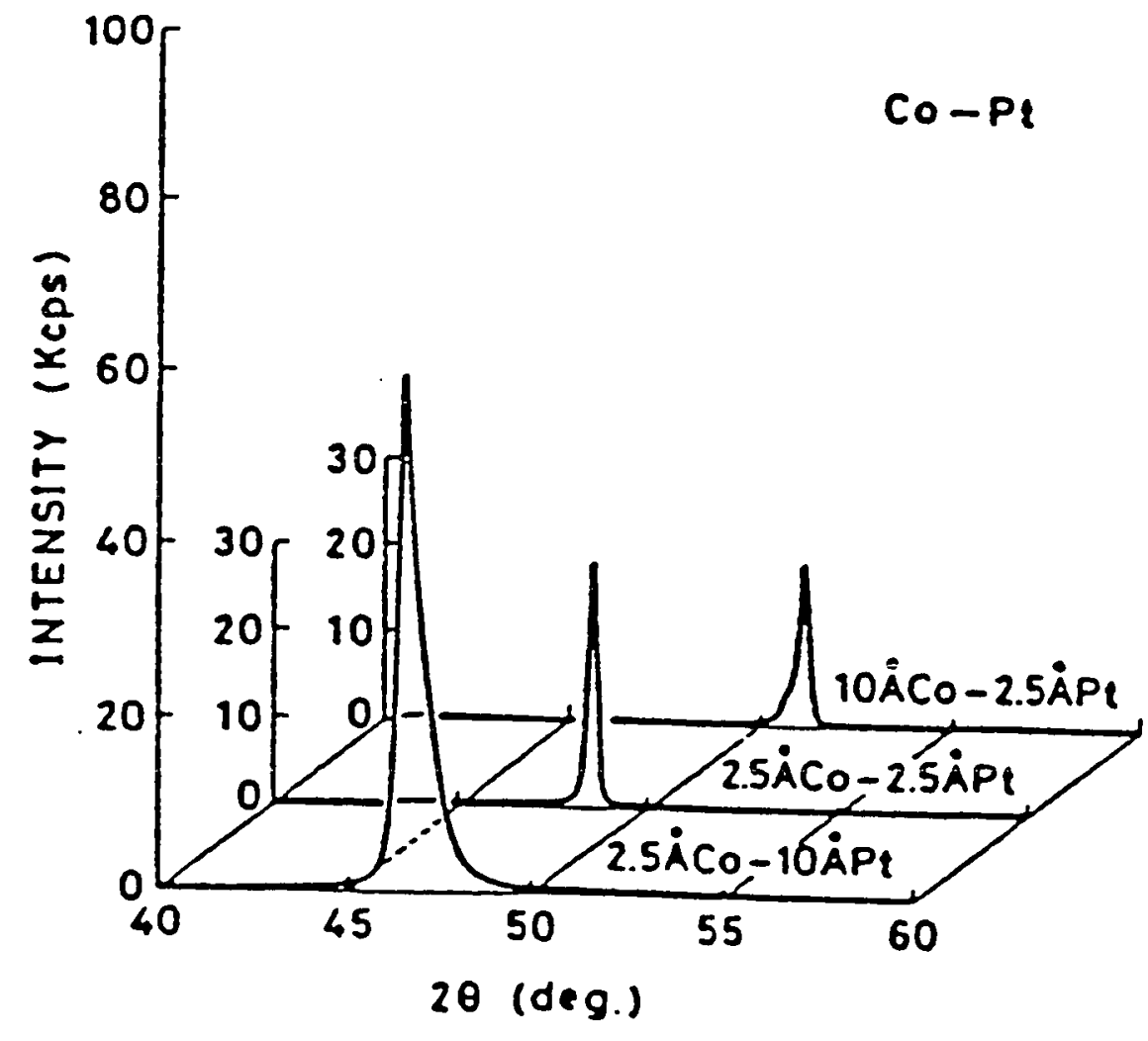

Figure 14. X-ray Diffraction Patterns for $1 \mathrm{~nm}$ Co and $.25 \mathrm{~nm} \mathrm{Pt}$ Layers, $.25 \mathrm{~nm} C o$ and
(Co $\mathrm{K}_{\infty}$ radiation). (32) $\mathrm{nm} \mathrm{Pt}$ Layers, and $.25 \mathrm{~nm} \mathrm{Co}_{0}$ and $1 \mathrm{~nm} \mathrm{Pt}$ Layers 


\begin{tabular}{|c|c|c|}
\hline \multirow[b]{2}{*}{$\begin{array}{c}\text { Co; hcp, } a=2.507 \dot{A} \\
c=4.07 \dot{A}\end{array}$} & \multicolumn{2}{|c|}{$\begin{array}{c}\mathrm{Pl} \\
\text { fcc; } a=3.9231 \AA\end{array}$} \\
\hline & $\begin{array}{c}\text { Normal } \\
\text { Co Pt } \\
\langle 001\rangle / /(111\rangle\end{array}$ & $\begin{array}{l}\text { Parallel } \\
\text { Co } \mathrm{Pl} \\
\langle 100) / /(110)\end{array}$ \\
\hline $\begin{array}{l}\text { Atomic distances }(\dot{A}) \\
\text { Difference in } \\
\text { atomic distances }(\dot{A}) \\
\text { Crystallographic } \\
\text { structure }\end{array}$ & $\begin{array}{c}\begin{array}{c}2.035,2.265 \\
-0.230\end{array} \\
\text { hep:Co layer }>\mathrm{Pt} \text { layer } \\
\text { fcc:Co layer < } \mathrm{Pt} \text { layer }\end{array}$ & $\begin{array}{c}2.507 .2 .774 \\
-0.267\end{array}$ \\
\hline Co $; f_{C c} a=3.5446 \dot{A}$ & $\begin{array}{c}\text { Normal } \\
(111) / /(111)\end{array}$ & $\begin{array}{c}\text { Parallel } \\
\langle 110\rangle / /(110\rangle)\end{array}$ \\
\hline $\begin{array}{l}\text { Atomic distances }(\dot{A}) \\
\text { Differcnce in } \\
\text { atomic distances }(\dot{A}) \\
\text { Crystallographic } \\
\text { structure }\end{array}$ & $\begin{array}{l}2.047,2.265 \\
-0.218 \\
\end{array}$ & $\begin{array}{c}2.506,2.774 \\
-0.268\end{array}$ \\
\hline
\end{tabular}

Table 1. Crystallographic data and differences in atomic distances for a multilayer structure. "Normal" and "Parallel" indicate the directions with respect to the film plane. "-n sign indicates that atomic distance of the Co layer is smaller than that of the Pt layer. The crystallographic structure closed with parentheses indicates an expected structure. (32) 


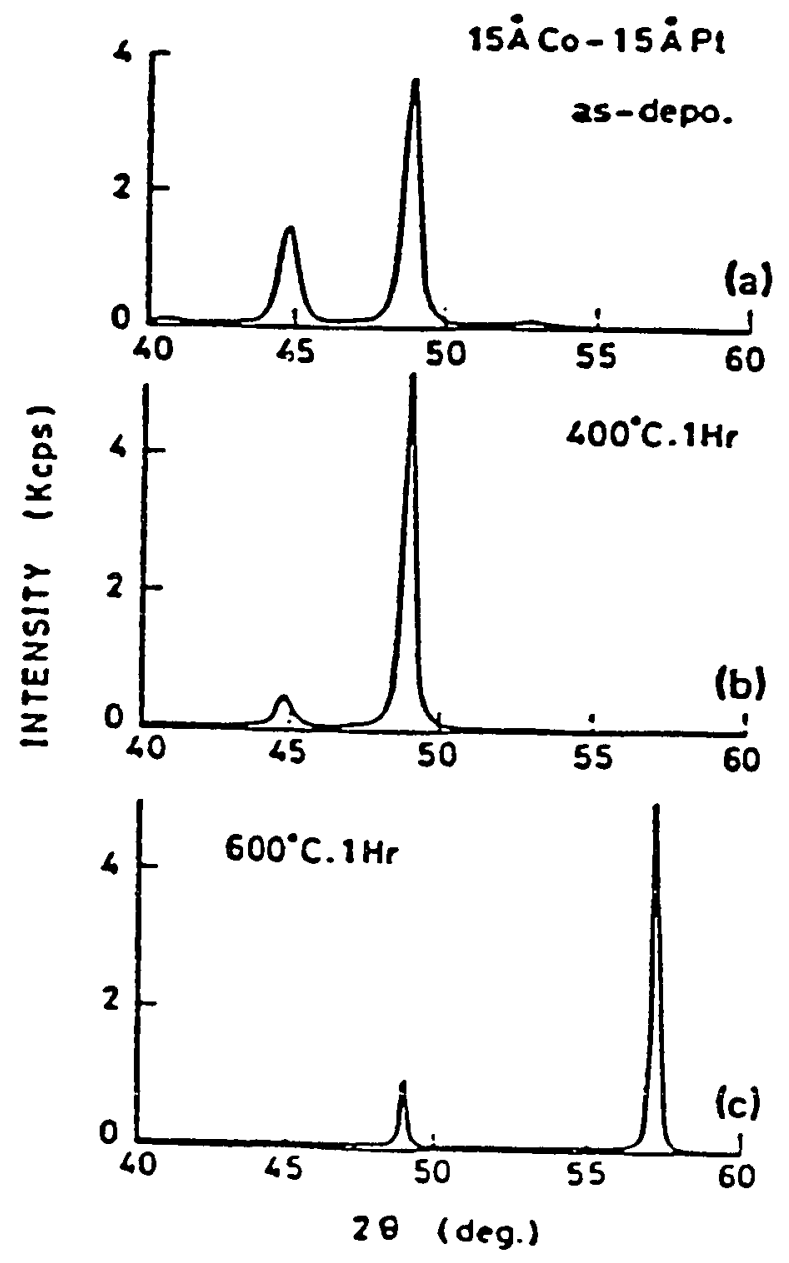

Figure 15. X-ray Diffraction Patterns of the Film with $1.5 \mathrm{~nm}$ Co and $1.5 \mathrm{~nm}$ Pt Layers: (a) As-Deposited, (b) After Annealing at $400^{\circ} \mathrm{C}$ for 1 Hour, (c)After Annealing at $600^{\circ} \mathrm{C}$ for 1 Hour. The Peak observed at $57^{\circ}$ on (c) is Identified as (110) Diffraction of the Co-Pt Alloy. (32) 
to the (002) diffraction of hcp-Co, are also present. In the film annealed at $400^{\circ} \mathrm{C}$ in 1 hour, the satellite peak intensities decrease, which indicates an interdiffusion at layer interfaces. When the annealing temperature increases to $600^{\circ} \mathrm{C}$, the film contains mostly Copt alloy, which is identified by the new diffraction peak at $57^{\circ}$ (tetragonal (110)) .

A perpendicular magnetization hysteresis loop with coercivity less than 1 Koe was observed in films with co thickness less than $1.5 \mathrm{~nm}$. The saturation magnetization of films is very close to the magnetization of bulk Co, which indicates Pt atoms in Co/Pt films did not have the induced magnetization. The author assumed that the perpendicular magnetic anisotropy of the multilayer films was caused by surface anisotropy at the Co/Pt interfaces.

In $1989 \mathrm{~W} . \mathrm{B}$. Zeper et al. (33) reported properties of improved co/Pt multilayer films which were made by the electron-beam evaporation technique. The films have $100 \%$ magnetic remanence and a square hysteresis loop when the co thiciness is 0.2 to $0.4 \mathrm{~nm}$. The coercivity of these films was also around 1 kOe. Recording performance testing was done on these films; a Carrier-to-Noise ratio (CNR) of $49 \mathrm{~dB}$ was reported. Although the CNR of these films is lower than 
those of RE-TM alloy, the results are very encouraging. If more research and development is conducted on the co/pt multilayer structures, better recording performance may be possible.

Review of the literature on Copt alloy and co/pt multilayer thin films indicates that the crystal structure and properties of the alloy films depend on the chemical composition of the film. For the case of the multilayer structures, the structure and properties of the film depend on the thickness of Co and Pt layers. The films deposited by e-beam evaporation technique [ref (34)] (33) have better properties than those made by the sputtering method [ref (20) ] . 
Chapter 4

EXXPERIMDHNTAL METHODS

\subsection{Experimental Rationale}

Based on the literature review, an experimental plan was designed:

- E-beam evaporation technique will be used rather than sputtering to deposit the films. It is chosen because the effect of particle bombardment, which is a feature of the sputtering method, is eliminated.

- Deposition rates will be kept as low as possible to allow the Co and Pt atoms enough time to occupy favored lattice sites.

- The effects of the Co and Pt thickness and the total thickness on the film properties and structure are to be examined.

\subsection{Film Deposition and Processing.}

Co/Pt multilayers were prepared in high vacuum by ebeam evaporation from separate Co and Pt sources onto 7059 Corning glass substrates. 
The e-beam evaporator consists of a stainless steel vacuum chamber which is evacuated by a cryopump. The base pressure of approximately $2 \times 10^{-8}$ Torr can be obtained after 10 hours of pumping. Inside the vacuum chamber, there are two electron guns which are mounted on the bottom of the chamber; one gun has one water-cooled copper crucible and the other has four. At the top center of the chamber, there is a combination heating and rotating substrate holder. Four quartz lamps of total 2000 watts of power are used to heat the substrates. The substrate temperature is monitored by a thermocouple placed between the lamps and the substrates.

Above the e-guns, shutters are used to shield the substrates from the particle flux during pre-deposition. Each shutter has one hole which allows vapor to reach the crystal rate monitor.

To produce Co/Pt multilayers, the evaporation rates of Co and Pt were kept at .02nm/sec and .05nm/sec, respectively, and the thickness of each layer was controlled by periodically opening and closing the shutters.

The evaporation rates of $\mathrm{Co}$ and $\mathrm{Pt}$ were calibrated by the following technique: 
A Co film, approximately $200 \mathrm{~nm}$ thick was deposited onto a glass substrate. The physical thickness $t_{\text {co }}$ of the film was measured by an Alfa step profilometer. The ratio $\mathrm{R}$ of the physical thickness and the thickness recorded by the thickness monitor, $t_{C o(m)}$, is used to calculate the real thickness of each co layer in the co/pt multilayer structure.

$$
t_{\mathrm{Co}}=\mathrm{R} \mathrm{t}_{\mathrm{Co}(\mathrm{m})}
$$

A similar technique was used to calibrate the Pt evaporation rate.

The modulation length, D, which is equal to the sum of the Co and Pt thickness, $t_{C o}+t_{P t}$, was measured by the small-angle x-ray diffraction method (see 4.3.3)

\subsection{Film Characterization.}

4.3.1 Kerr Hysteresis Looper with Heating Stage.

A MO Kerr hysteresis looper was used to measure the polar Kerr rotation angle, coercivity and their temperature dependencies (22). As shown in Fig. 16, Iinearly polarized light is incident upon a sample held to a sample holder by vacuum. The sample holder is equipped with a heating stage 
(designed and built by the author) capable of raising the temperature of the sample to $450^{\circ} \mathrm{C}$. The entire sample holder is positioned between pole pieces of an electromagnet where a field of up to 18 koe can be obtained at the sample. The reflected light is transmitted through a Wollaston polarizing beam splitter. The two split beams are sensed by two photodetectors with identical gains. Then, by using

$$
\tan 2 \theta_{k}=I_{1} \times I_{2} / I_{1}+I_{2} \quad \text { eg. (5) }
$$

where $I_{1}$ and $I_{2}$ are the light intensity of the two split beams, $\theta_{k}$ can be derived. A GaAs, HeNe, or Kr laser is selectively used as a probe beam for different wavelength measurements $(\lambda=820,633$ and $488 \mathrm{~nm}$, respectively). An IBM PCAT is used to control the loop tracing, data acquisition, and data analysis.

4.3.2 Vibrating Sample Magnetometer (VSM) .

The magnetization of the Co/Pt multilayer films used in this study was measured by a vibrating sample magnetometer (VSM). The simplified block diagram of the VSM is shown in fig. 17 (22)

When a specimen is placed in the uniform magnetic field (provided by the electromagnet), a dipole moment 


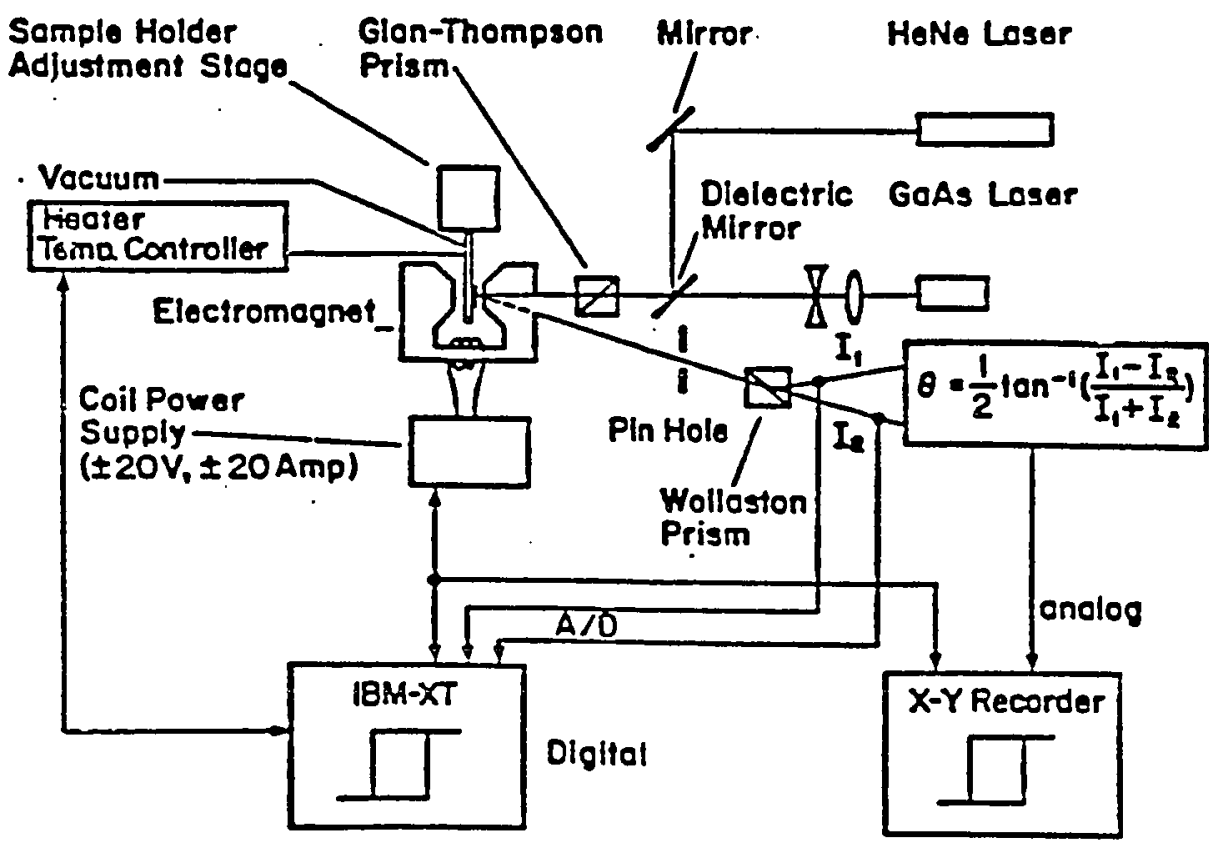

Figure 16. Schematic Diagram of a MO Kerr Hysteresis Looper.

(22) 
proportional to the product of the sample susceptibility times the applied field is induced in the sample. If the sample is made to oscillate as well, an electrical signal can be induced in the pick-up coils mounted on both sides of the magnet pole pieces. The signal, which is at the oscillation frequency, is proportional to the magnetic moment, vibration amplitude and vibration frequency. The reference signal is provided by the transducer. The dc voltage, proportional to the magnetic moment but independent of changes in vibration amplitude and frequency, can be derived from the signal processing circuitry as depicted in Fig. 17

4.3.3 X-Ray Diffractometer.

Crystallographic structures of films were examined by standard $x$-ray diffraction with $\mathrm{Cu}$ Ka radiation.

The modulation lengths $D$ were measured by small-angle $x$-ray diffraction $\left(\theta<10^{\circ}\right)$. This method is based on the direct scattering by the difference in $x$-ray refractive index of the two constituents (34).

$$
D=\lambda / 2\left(\sin \theta_{i}-\sin \theta_{i+1}\right)
$$




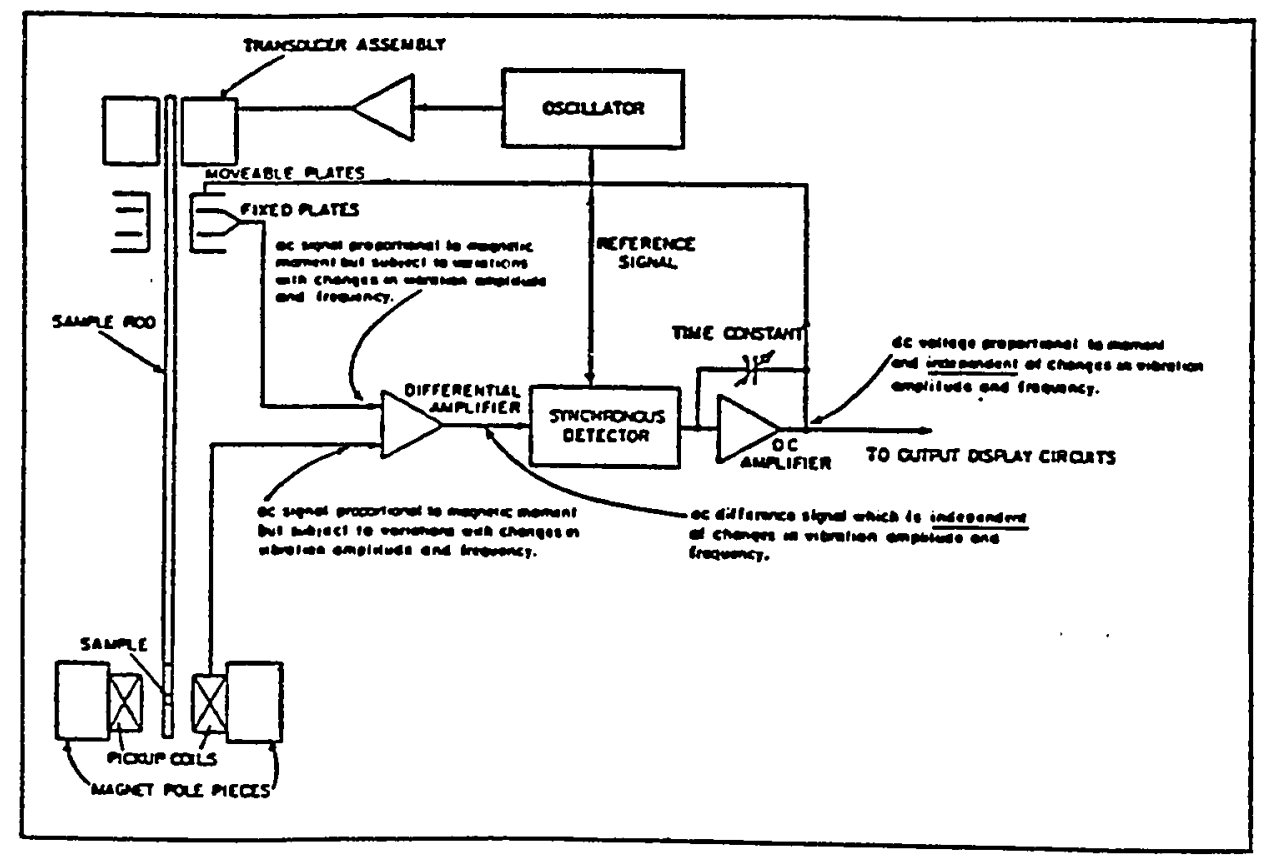

(22)

Figure 17. Schematic Diagram of a Vibrating Sample Magnetometer (VSM). 
where $\lambda$ is the $x$-ray wavelength, and $i$ and $i+1$ refer to the adjacent diffraction peaks in the low-angle x-ray diffraction spectrum. In $f i g .18$, if the $\theta_{i+1}$ is equal $6.7^{\circ}$ then the calculated $D$ is:

$$
\begin{aligned}
& D=.154 \mathrm{~nm} / 2\left(\sin 6.7^{\circ}\right) \\
& D=1.31 \mathrm{~nm}
\end{aligned}
$$

The value of $D$ is very close to the sum of $t_{C o}$ and $t_{P t}$ calculated from the deposition rates $(1.31 \mathrm{~nm}$ as compared to $0.3 \mathrm{~nm}+1.0 \mathrm{~nm}$ determined from the deposition date). 


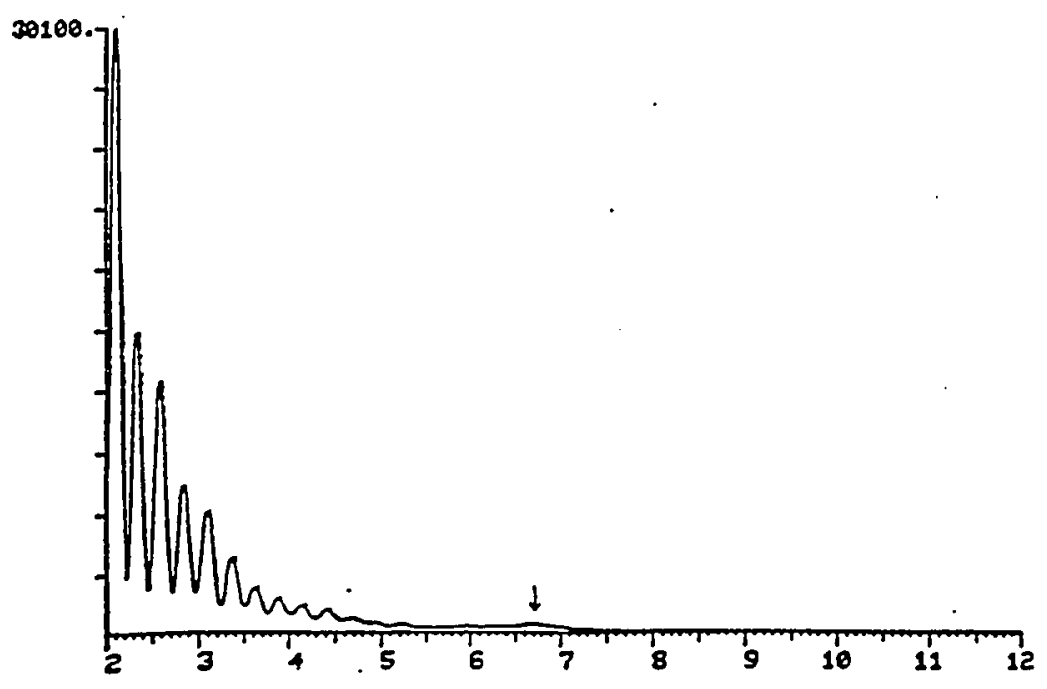

Figure 18. Low-angle $\mathrm{X}$-ray Diffraction Pattern of the As-deposited Co $(0.3 \mathrm{sm}) / \mathrm{Pt}(1 \mathrm{~nm})$ Multilayer Film. (CuK $\alpha$ radiation). 


\section{Chapter 5}

\section{RESULTS AND DISCUSSIONS}

Because some of data obtained for the Co/pt multilayer films have become IBM confidential, only the effects of Co thickness, Pt thickness, and the total thickness of the multilayer films on their properties and structure are discussed herein. The results are tabulated in Table 2.

Table 2 summarizes the magnetic and magneto-optical properties of the Co/Pt multilayer films which were deducted from the MO hysteresis loops.

\subsection{MO Hysteresis Loop Characteristics}

The effects of Co and Pt thicknesses on the shape of the Kerr hysteresis loop are summarized in Fig. 19 and the variation in Kerr rotation angle $\theta_{k}$, coercivity $\mathrm{H}_{C^{\prime}}$, and nucleation field $\mathrm{H}_{\mathrm{n}}$ as a function of Co layer thickness are shown in Figs. 20 and 21 .

The coercivity $\mathrm{H}_{\mathrm{C}}$ and the nucleation field $\mathrm{H}_{\mathrm{n}}$ strongly depend on the thickness of the Co layers, $t_{\text {Co. }}$. For the constant Pt thickness of $1 \mathrm{~nm}$, the $H_{C}$ and $H_{n}$ have $h i g h$ 
Table 2. Constitution, magnetic and MO properties of Co/Pt multilayer structures. $t_{\mathrm{Co}}$ is the Co thickness, $t_{\mathrm{Pt}}$ is the Pt thickness, $\theta_{\mathrm{K}}$ is the Kerr rotation angle (saturation value). $\mathrm{H}_{\mathrm{C}}$ is the coercivity, $\mathrm{Hn}_{\mathrm{n}}$ is the nucleation field, and $T_{C}$ is the Curie temperature. The films are asdeposited.

\begin{tabular}{|c|c|c|c|c|c|c|c|}
\hline Sample & $\begin{array}{l}t_{c o} \\
(\mathrm{~m})\end{array}$ & $\begin{array}{l}t_{p t} \\
(m i n)\end{array}$ & ${ }_{(\mathrm{m})}^{\mathrm{t}_{\text {Tot }}}$ & $\begin{array}{c}\theta_{K} \\
\text { (degree) }\end{array}$ & $\begin{array}{c}H_{c} \\
(K O E)\end{array}$ & $\begin{array}{r}H_{n} \\
(K D e)\end{array}$ & $\begin{array}{l}T^{\top} c \\
\left.{ }^{0} c\right)\end{array}$ \\
\hline 1 & 0.1 & 1.0 & 30.0 & 0.07 & 0.3 & 0.2 & \\
\hline 2 & 0.2 & 1.0 & 30.0 & 0.13 & 1.8 & 1.8 & \\
\hline 3 & 0.3 & 1.0 & 30.0 & 0.20 & 1.9 & 1.9 & 205 \\
\hline 4 & 0.4 & 1.0 & 30.0 & 0.22 & 1.2 & 0.9 & 325 \\
\hline 5 & 0.5 & 1.0 & 30.0 & 0.21 & 0.7 & -0.4 & \\
\hline$s$ & 0.6 & 1.0 & 30.0 & 0.24 & 0.6 & -3.0 & 425 \\
\hline 7 & 0.7 & 1.0 & 30.0 & 0.25 & 0.5 & -4.0 & \\
\hline 8 & 0.3 & 0.4 & 30.0 & 0.24 & 1.3 & 0.5 & \\
\hline 9 & 0.3 & 0.8 & 30.0 & 0.18 & 1.7 & 1.7 & \\
\hline 10 & 0.3 & 1.5 & 3.0 & 0.12 & 1.4 & 1.4 & \\
\hline$n$ & 0.3 & 1.0 & 10.0 & * & 1.9 & 1.9 & \\
\hline 12 & 0.3 & 1.0 & 20.0 & $*$ & 1.9 & 1.9 & \\
\hline 13 & 0.3 & 1.0 & 30.0 & 0.20 & 1.9 & 1.5 & 205 \\
\hline 14 & 0.3 & 1.0 & $50 . c$ & 0.20 & 2.2 & 1.9 & 295 \\
\hline 15 & 0.3 & 1.0 & 100.0 & 0.20 & 3.6 & 1.9 & 205 \\
\hline 16 & 0.1 & 0.2 & 30.0 & 0.12 & 1.2 & 0.9 & \\
\hline 17 & 2.0 & 2.0 & 30.0 & 0.17 & $0 . n$ & -6.0 & \\
\hline
\end{tabular}

* For films thinner than $30 \mathrm{~nm}$, optical interferences alters the $\theta_{\mathrm{K}}$ value.

** $\theta_{\mathrm{K}}$ is characterized at HeNe laser wavelength (633nm)

*** The film substrate is Corning 7059 glass 


\begin{tabular}{|c|c|c|c|c|c|c|c|c|}
\hline${ }_{6}$ & $4 \stackrel{\circ}{4}$ & $5 i^{\circ}$ & $\therefore i$ & $7 \AA$ & $\therefore$ & $10 \AA^{\circ}$ & $13 \AA^{\circ}$ & $20 \AA^{\circ}$ \\
\hline $2 \AA^{\circ}$ & $\sqrt{T}$ & & & & & & & J \\
\hline $3 i^{\circ}$ & $\pi$ & & & & & & & $\square$ \\
\hline $4 \AA^{\circ}$ & $\sqrt{8}$ & $\Gamma$ & & $\int$ & & & & \\
\hline si & & & & & & & & \\
\hline $6 i$ & & & $\mathbb{J}$ & & & & & \\
\hline $7 i$ & & & & & & & & \\
\hline$\stackrel{\circ}{\circ}$ & & & & & & & & \\
\hline
\end{tabular}

Figure 19. The Effects of Co and Pt Thicknesses on the Shape (degree of squareness) of the Kerr Hysteresis Loop. 


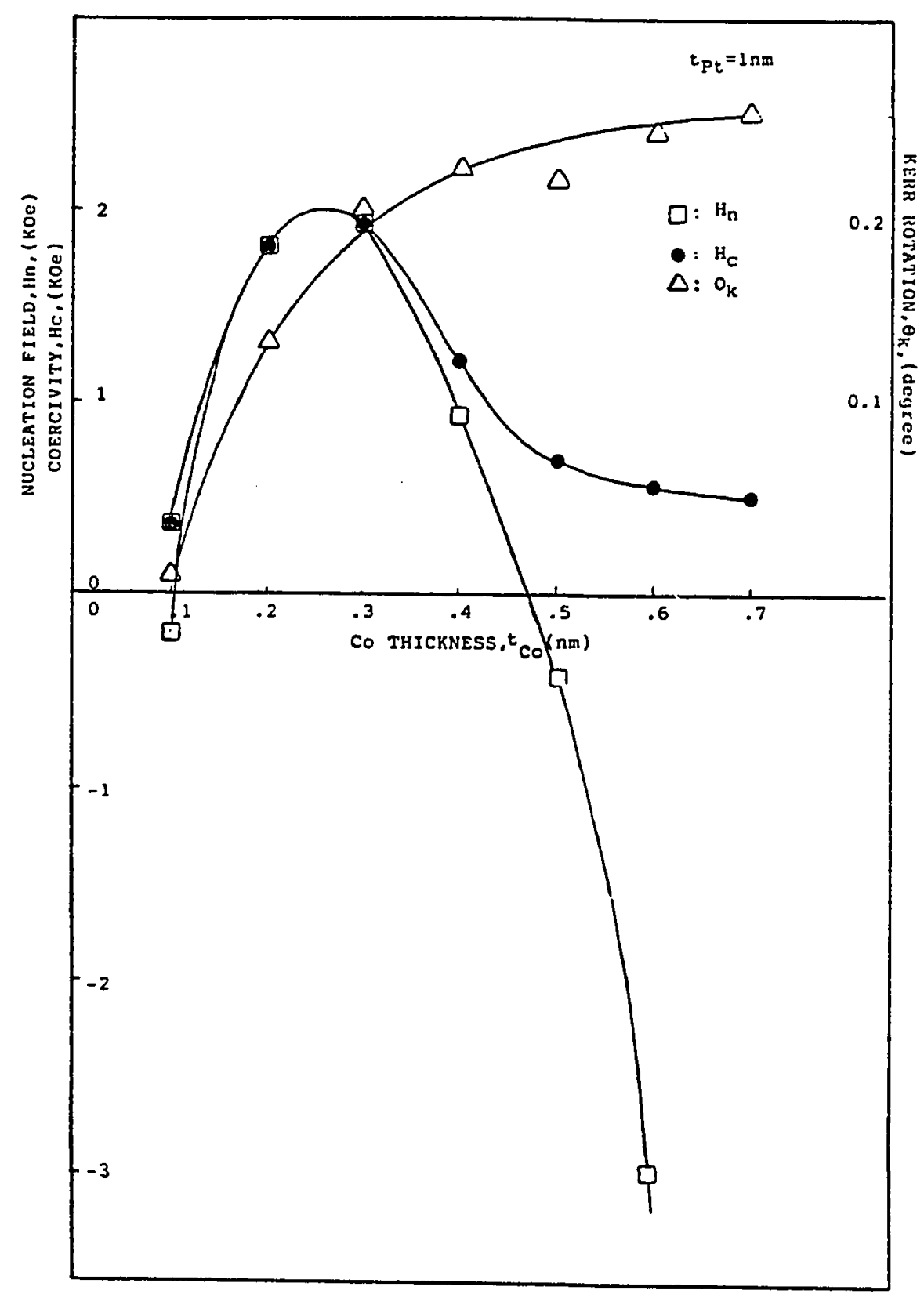

Figure 20. Kerr Rotation $\theta_{k}$, Coercivity $\mathrm{H}_{c}$, and Nucleation Field $\mathrm{H}_{n}$, vs the Thickness of Co Layer in As-deposited Co/Pt Multilayer Films. 


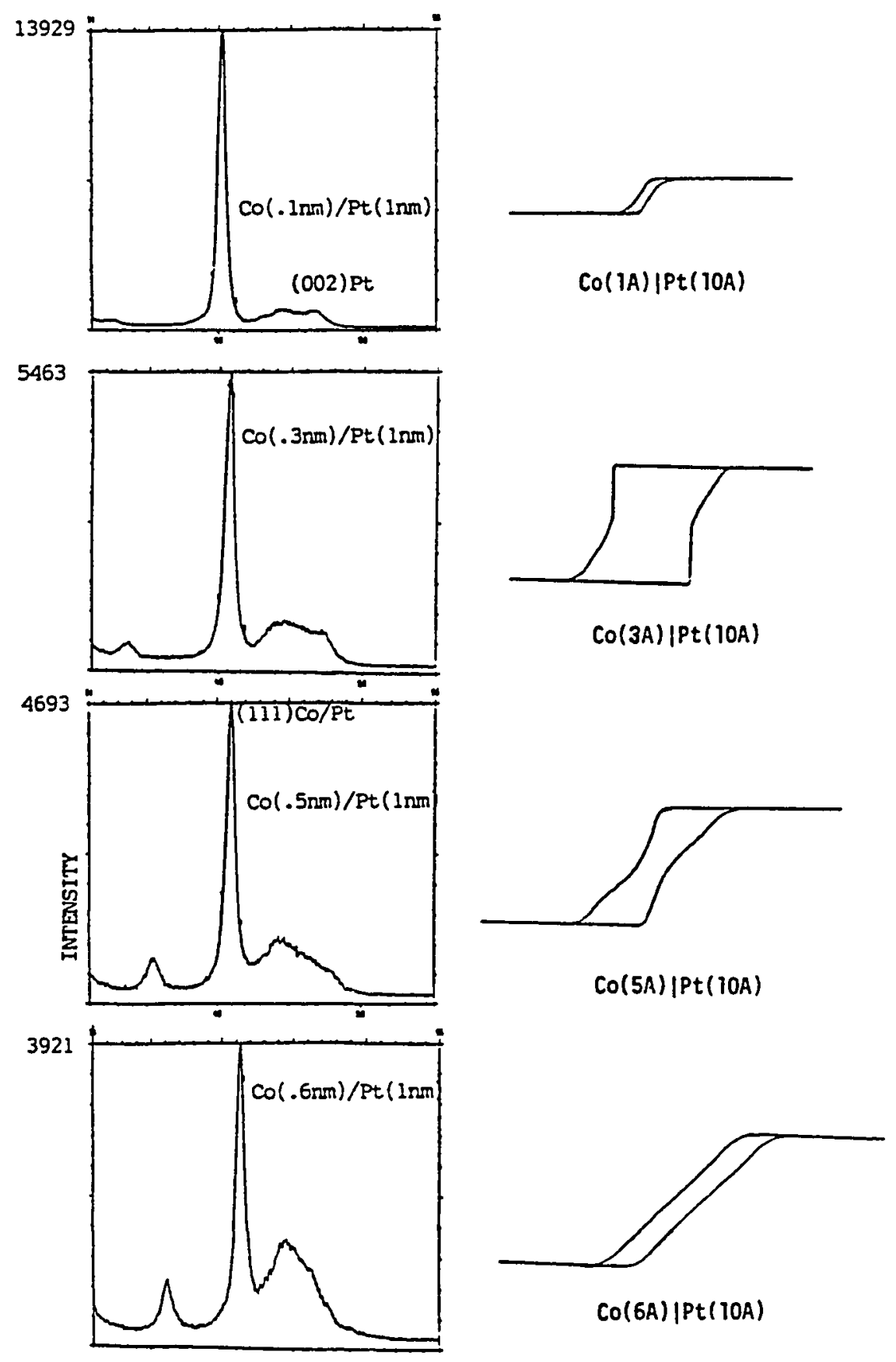

Figure 21. Kerr Loops of Co/Pt Multilayers vs Co Thickness. 
values ( $1.9 \mathrm{KOe})$ when the thickness of Co layers is 0.2 to $0.3 \mathrm{~nm}$. As the Co thickness increases above $0.4 \mathrm{~nm}, \mathrm{H}_{\mathrm{n}}$ and $H_{C}$ decrease sharply. $H_{n}$ becomes negative when $t_{C o}$ is equal to or greater than $0.5 \mathrm{~nm}$.

when the co thickness is constant at $0.3 \mathrm{~nm}$, the coercivity $\mathrm{H}_{C}$ of the multilayer films depends weakly on the thickness of the Pt layers (refer to Table 2). Fig. 22 shows that when the Pt thickness is small $(0.4 \mathrm{~nm})$, the Kerr loop becomes more sheared. This may indicate that the thin Pt layer $(0.4 \mathrm{~nm})$ has a different crystallographic structure than the thicker one $(1 \mathrm{~nm})$; and the Pt layer structure may influence the formation of the subsequent co layer.

\section{$\underline{5.2 \quad \text { Structures }}$}

X-ray diffraction patterns representing multilayer films with different Co thickness, Pt thickness and total film thickness are shown in Figs. 23, 24, and 25 , respectively. The standard $x$-ray diffraction spectrum of pure Pt, pure Co, and CoPt (50:50 at. percent) alloy, are included in Fig. 26 for comparison purposes.

The appearance of the satellite peaks (see fig. 23, for example) around the mean peak in most of the $x-r a y$ 

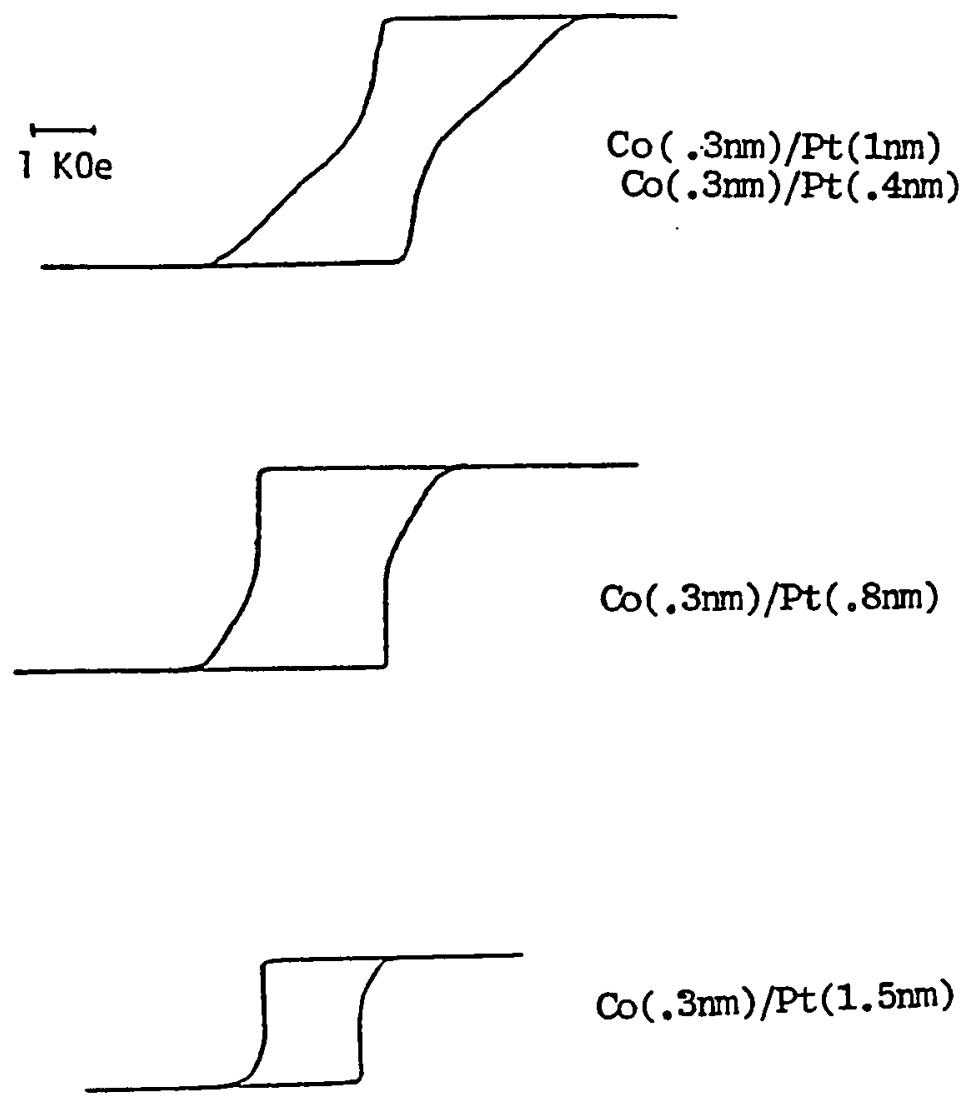

Figure 22. Kerr Loop of Co/Pt Multilayers vs Pt Thickness. 

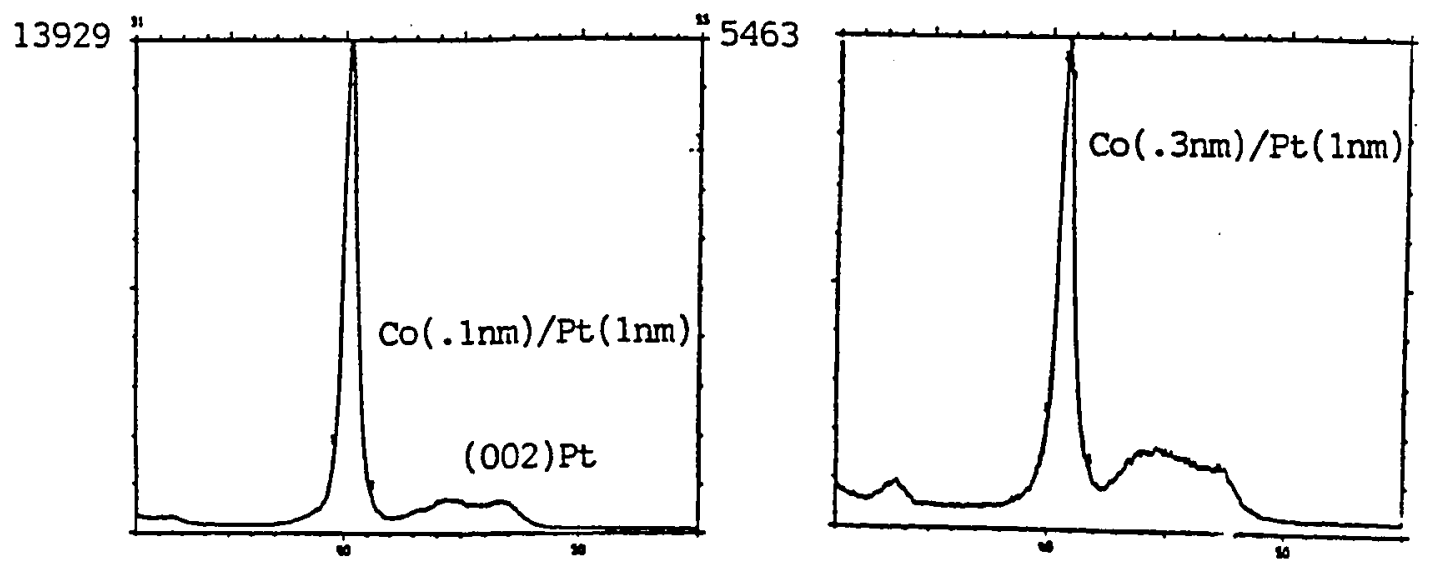

4693
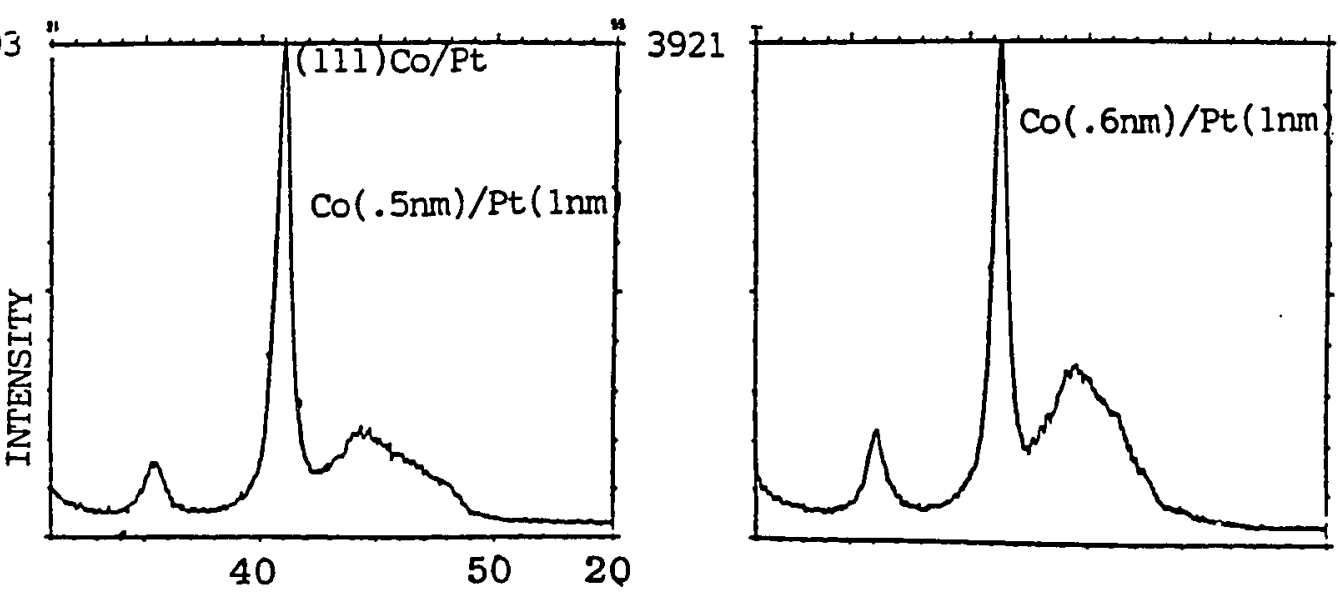

Figure 23. X-ray Diffraction Spectrum of Co/Pt Multilayer Films vs Co Thickness. 


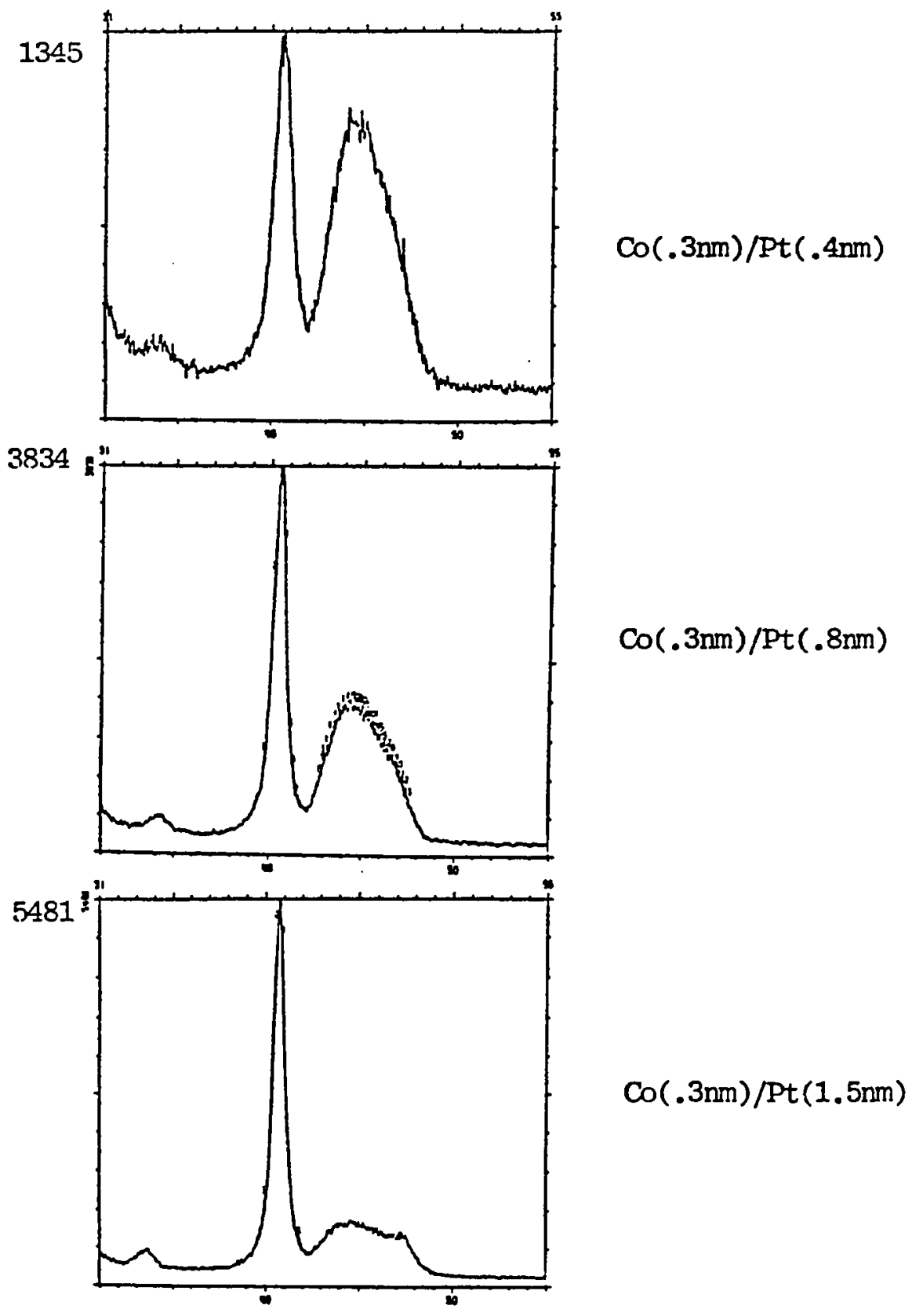
Figure 24. X-ray Diffraction Spectrum of Co/Pt Multilayer Films vs Pt
Thickness. 


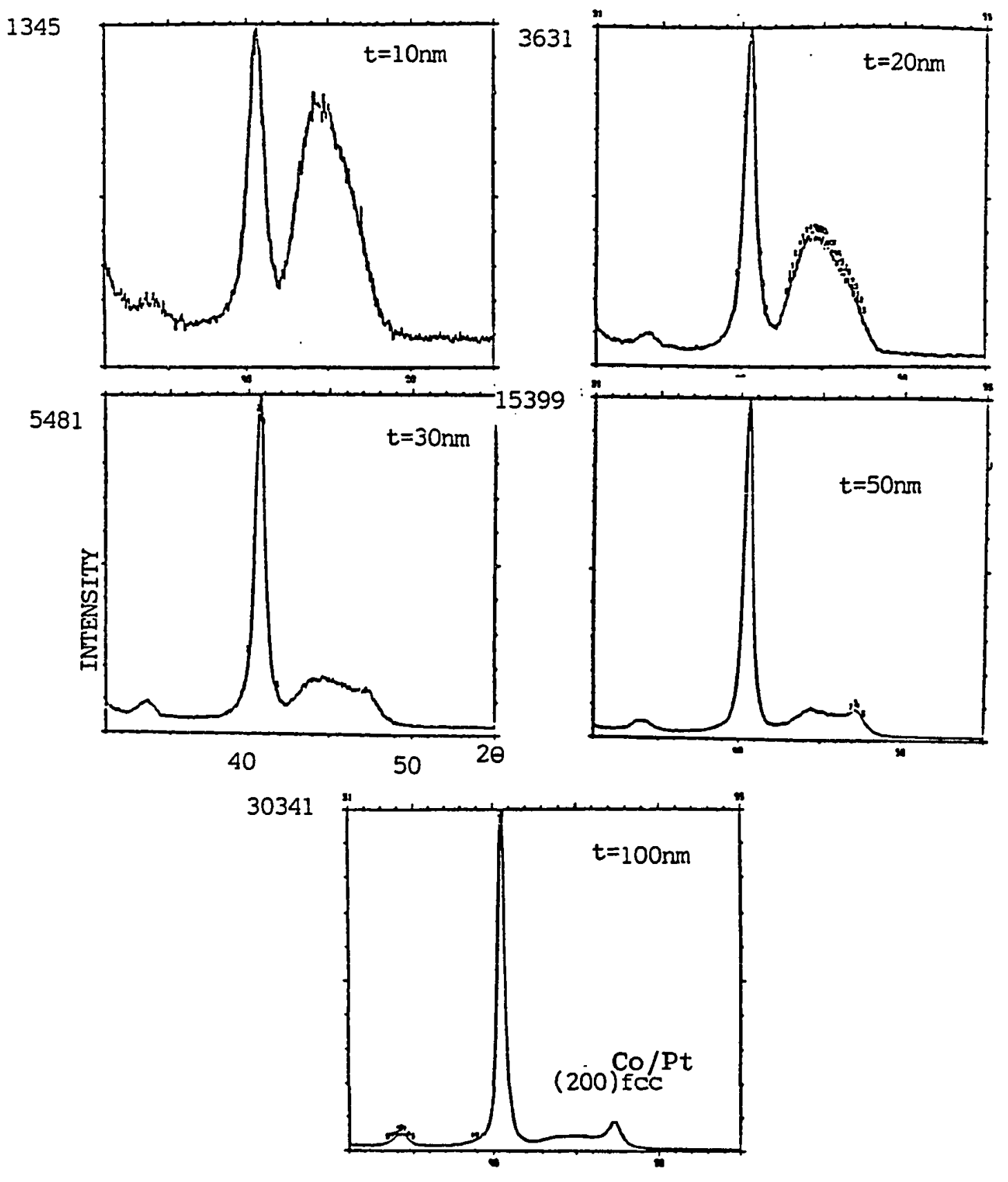

Figure 25. X-ray Diffraction Spectrum of Co/Pt Multilayer Films vs Total Film Thickness. 


\section{$\operatorname{Co}(.3 \mathrm{~nm}) / \mathrm{Pt}(1 \mathrm{~nm})$}
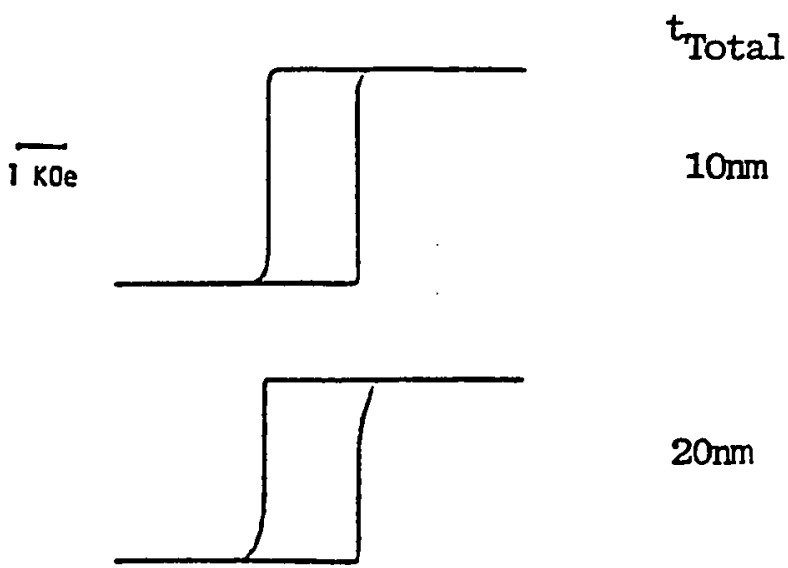

$20 \mathrm{~nm}$

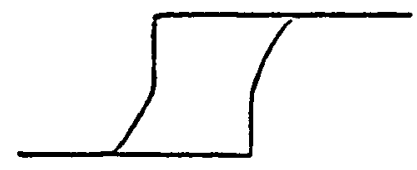

$30 \mathrm{~mm}$

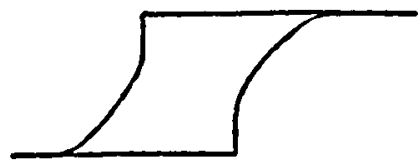

$50 \mathrm{~nm}$

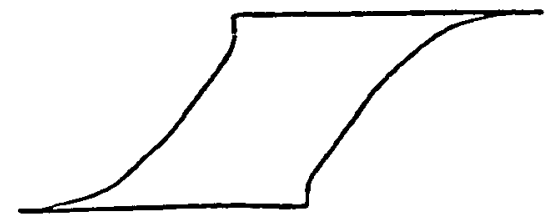

$100 \mathrm{~nm}$

Figure 31. Kerr Loop vs Total Film Thickness. 


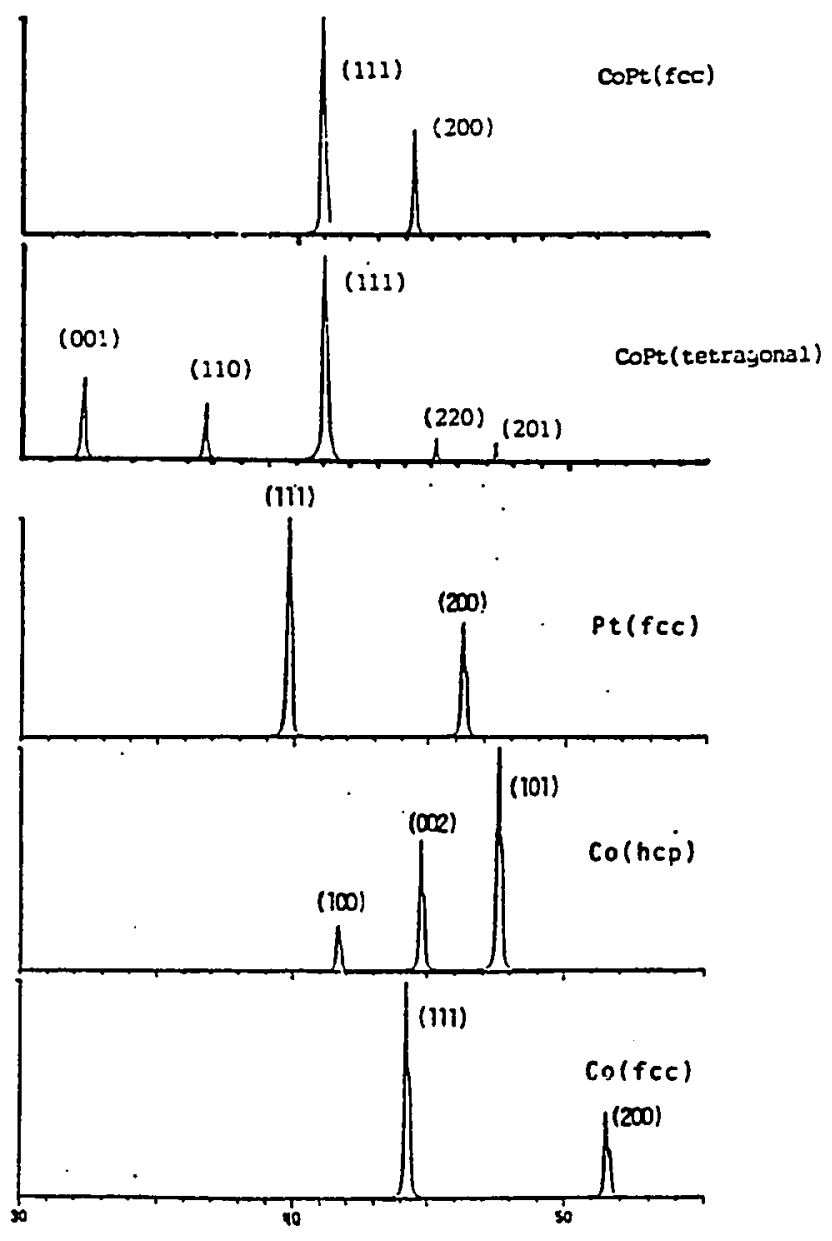

Figure 26. Standard $x$-ray Diffraction spectrum of $P t, C o$, and COPt (50:50 at. percent) thin films. 
diffraction spectra indicates the existence of a layered structure (see Figs. 22.c and 22.d). The highest intensity peak is the Copt multilayer peak which is between the fcc Pt (111) and the hcp Co (002) or fcc Co (111) satellite peaks. In Fig. 23(a), the intensities of the satellite peaks are very low. This indicates that the co layers are discontinuous, and the co atoms distribute in the Pt matrix. When the thickness of $C o$ and Pt layers increases, the layered structure is indicated clearly in the $x-r a y$ diffraction spectrum. The intensity of the satellite peaks increases sharply as is seen in Fig. 23 (d).

When the co thickness of the Co/Pt multilayer films (with constant Pt thickness at $1 \mathrm{~nm}$ ) increases (see Fig. 23) the mean peak position shifts to higher 20 angle (to the right). This also occurs when the Pt layer thickness decreases (Fig. 24). This indicates that the d spacing decreases when the concentration of co in the copt multilayer film increases.

For Co $(0.3 \mathrm{~nm}) / \mathrm{Pt}(1 \mathrm{~nm})$ multilayers, when the total film thickness increases, the intensity of hcp co (002) (or fcc Co (111)) decreases and the Co/Pt (200) peak intensity increases. This is consistent with a decrease in the perpendicular magnetic anisotropy in the film (see Fig. 25). 


\subsection{Magnetic Anisotropy}

Perpendicular magnetic anisotropy is an essential property for a magneto-optical recording medium. A magnetic thin film has high perpendicular magnetic anisotropy when all magnetic moments can be aligned perpendicular to the film plane easily. This indicates the film hard-axis to be in the film plane. The perpendicular magnetic anisotropy property of a magnetic thin film can be indicated by the shape of the Kerr hysteresis loop ( the relative values of $\mathrm{H}_{\mathrm{n}}$ and $\mathrm{H}_{\mathrm{C}}$ ). A film has high perpendicular anisotropy when its nucleation field $H_{n}$ value is very close to its coercivity $\mathrm{H}_{\mathrm{C}}$ value.

The magnetic hysteresis loop determines the orientation of the magnetization, $M$, of the film in more detail than the Kerr hyteresis loop. As seen in Fig. 27, the Col0.3 $\mathrm{nm}) / \mathrm{Pt}(1 \mathrm{~nm})$ multilayer film has a high perpendicular magnetic anisotropy; the in-plane magnetization component is near zero. On the other hand, the Co(2 $\mathrm{nm}) / \mathrm{Pt}(2 \mathrm{~nm})$ 

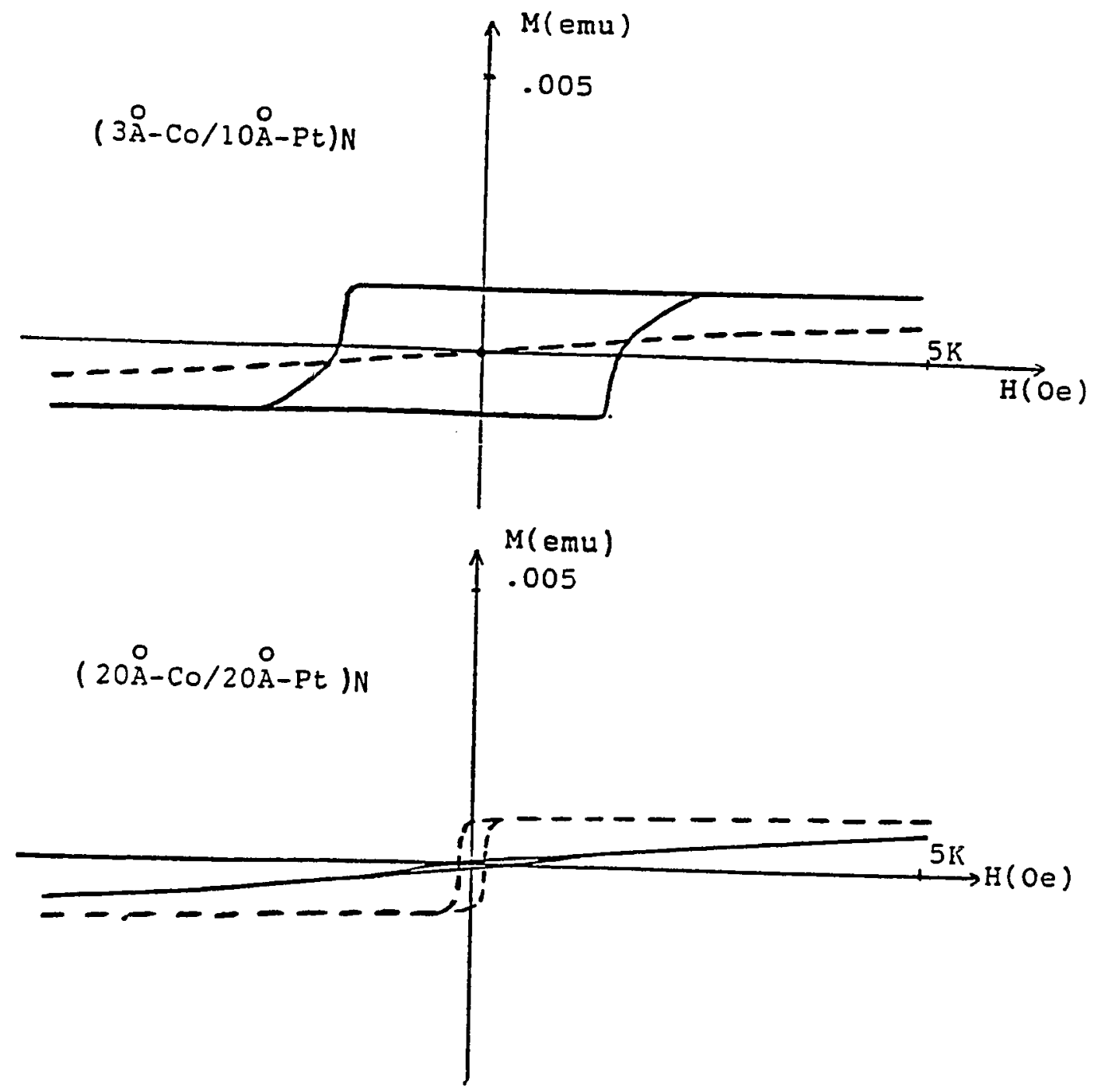

Figure 27. Magnetic Hysteresis Loops of Co/Pt Multilayer Films with Applied Magnetic Field in the Film plane (dashed curve) and Perpendicular to the Film (solid curve). 
multilayer film has a higher in-plane magnetization component than the perpendicular.

At constant Pt thickness (1 nm) and co thickness varying from 0.1 to $0.7 \mathrm{~nm}$, the Co/Pt multilayer structures have high perpendicular anisotropy when the co thickness varies from 0.1 to $0.4 \mathrm{~nm}$ (see Fig. 20). The $\mathrm{H}_{\mathrm{n}}$ value of these films is almost equal to the $\mathrm{H}_{C}$ value. When the Co thickness increases the Mo hysteresis loops become more sheared or harder to magnetize perpendicular to the film plane (refer to Figs. 20 and 21). The $H_{n}$ values of these films are negative as is seen in Fig. 21 .

It is rationalized that for the films with $0.1 \mathrm{~nm}$ co thickness, the co layers become discontinuous or islandlike, and the co atoms distribute in the Pt matrix. Co and Pt atoms do not alloy in the appropriate ratio to form a good ferromagnetic material. This leads to a small Kerr loop with small perpendicular magnetic anisotropy.

For the case of films with co layer thickness varying from 0.2 to $0.4 \mathrm{~nm}(1-2$ atomic layers), probably most of the Co atoms alloy with Pt atoms in the appropriate ratio to form high anisotropic ferromagnetic CoPt layers which are sandwitched by the Pt layers. 
When the thickness of the co layers is larger than 0.4 nm (more than two atomic layer) the co atoms at co-pt interfaces may alloy with pt atoms to form copt ferromagnetic layers. The rest of co atoms may have inplane magnetization which alters the magnetic properties of the Copt alloy layers and reduce the perpendicular magnetic anisotropy of the multilayer films.

The total thickness of the co/pt multilayer films has some effect on the perpendicular magnetic anisotropy. Fig. 25 show that when the total film thickness increases, the saturation field $\mathrm{H}_{S}$ increases. This may mean that the film contains domains which are not perpendicular to the film plane. $\mathrm{X}$-ray diffraction data support this hypothesis. $\mathrm{X}$ ray diffraction spectra of the $100 \mathrm{~nm}$ thick film (Fig. 25) show the intensity of the peak at $47.5^{\circ}$ to increase as the total film thickness increases. This peak is likely the (200) peak for fcc Co/Pt. This indicates that there is (200) orientation of fcc Co/Pt in the Co(0.3 nm)/Pt(1 nm) multilayer structures; and when the number of layers of this film increases, the effect of this orientation on the film magnetic anisotropy becomes stronger. 


\section{$\underline{5.4 \quad \text { Kerx Rotation, } \Theta_{k}}$}

The Kerr rotation angle of the Co/Pt multilayer films increases (a) as the Co thickness increases (for constant Pt thickness) or (b) as the Pt thickness decreases at constant $t_{\text {Co }}\left(\right.$ see Fig. 28). That means the $\theta_{k}$ in general increases when the concentration of co in the multilayer film increases. Kerr rotation is proportional to the saturation magnetization of the film. The saturation magnetization arises from the ferromagnetic Co atoms (see Fig. 20). But the Curie temperature also increases as the amount of Co in the film increases (see Fig. 29). The MO film which has the higher Curie temperature requires a higher laser power and thus becomes unsuitable for Mo recording application. Thus a balance of the Co:Pt ratio is important.

\subsection{Corrosion Resistance}

Samples $1,2,3,7,11,16$, and 17 were immersed in water for 30 days. These films did not corrode. The magnetic and magneto-optical properties of these film remained unchanged. 


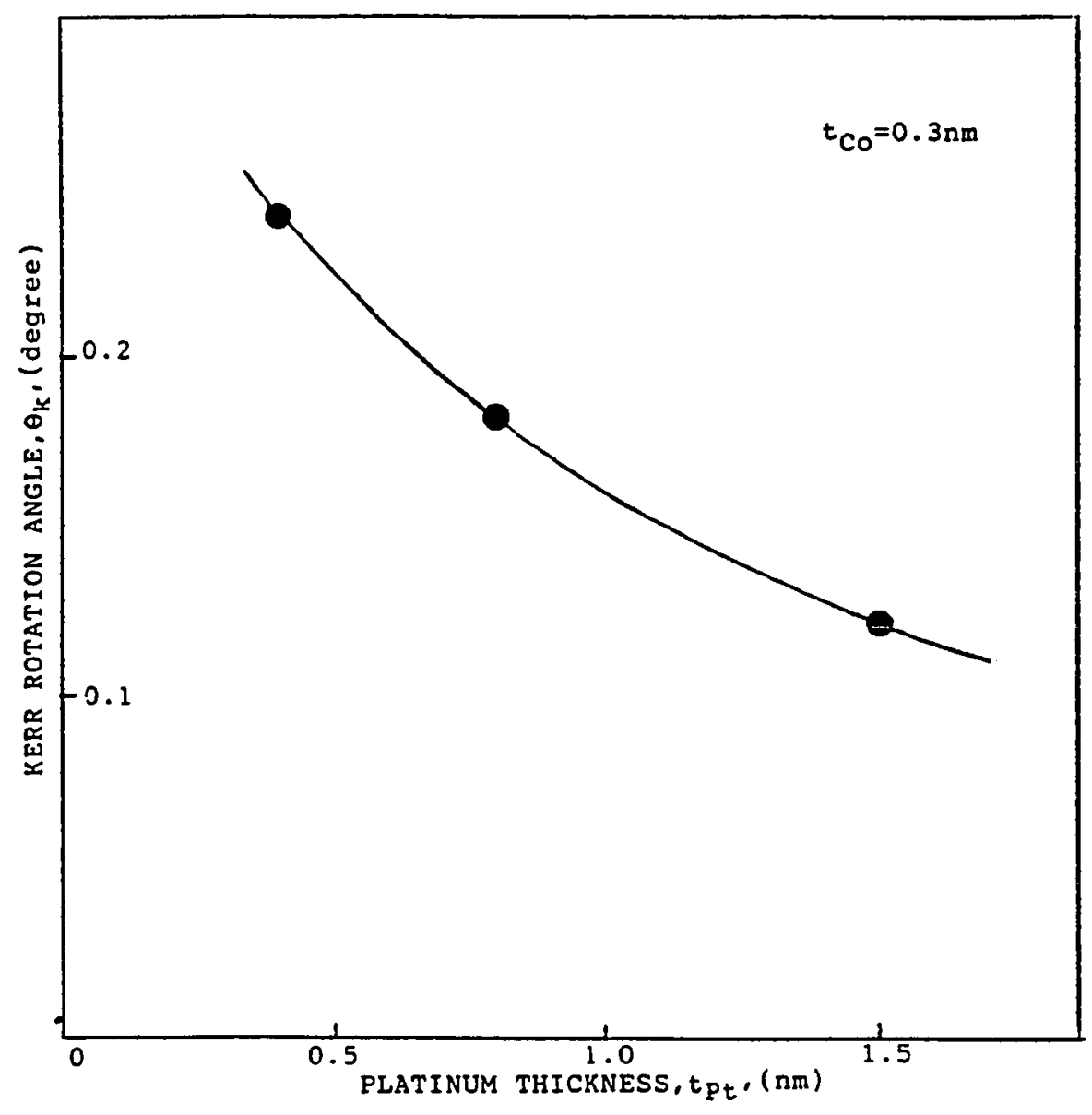

Figure 28. Kerr Rotation vs Pt Thickness. 


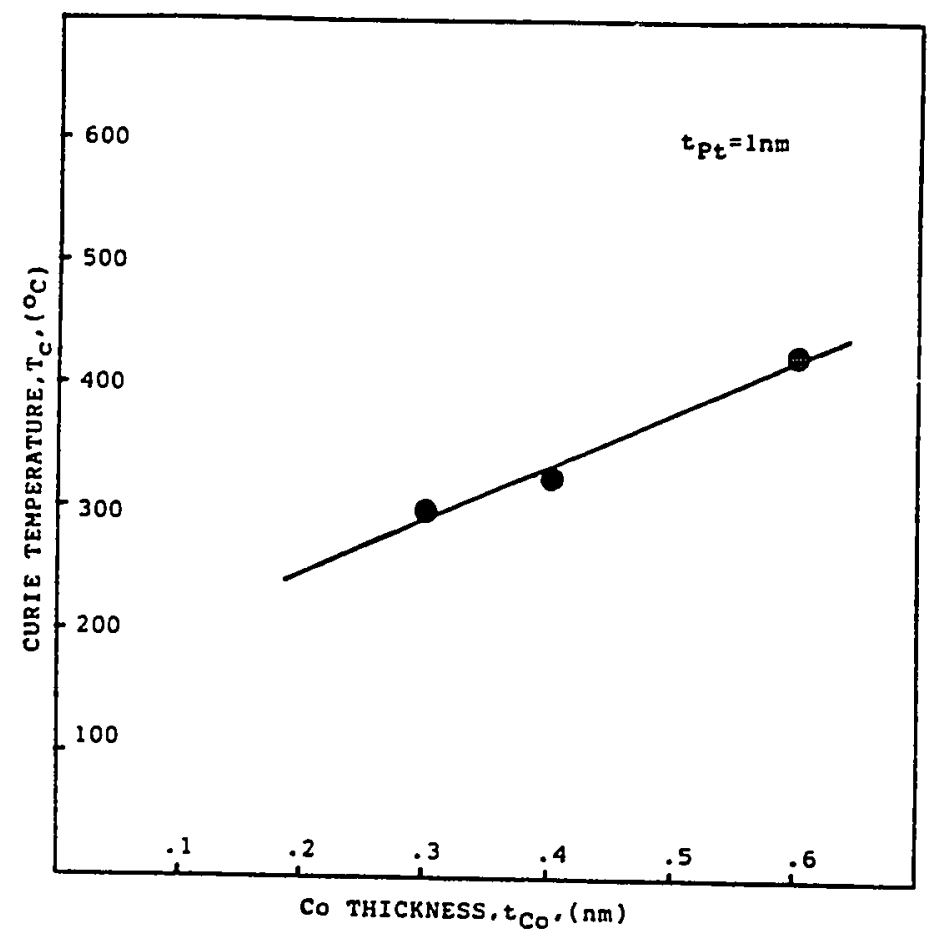

Figure 29. Curie Temperature vs Co Layer Thickness.

71 


\subsection{Effects Of Laser wavelengths On Kerr Rotation}

Lasers with wavelengths of 820,633 , and $488 \mathrm{~nm}$ (GaAs, HeNe, and $\mathrm{Kr}$, respectively) were used to examine the effect of the wavelength on $\theta_{k}$ Kerr rotation of a Co $(.3 \mathrm{~nm}) / \operatorname{Pt}(1$ nm) multilayer film. As is shown in Fig. 30, the rotation angle increases when the wavelength decreases from 820 to $488 \mathrm{~nm}$. This may provide an advantage for Co/Pt multilayer structures over RE-TM alloys for future Mo recording applications.

\subsection{Thermal stability}

Sample 3 of Table 2 was annealed in atmosphere at $350^{\circ} \mathrm{C}$ for 10 minutes. The annealed Co $(0.3 \mathrm{~nm}) / \mathrm{Pt}(1 \mathrm{~nm})$ multilayer film still has high perpendicular magnetic anisotropy. The $H_{C}$ decreased $10 \%$ and the $H_{n}$ value decreased $10 \%$. The Kerr rotation angle remains unchanged.

The high thermal stability of the co/pt multilayer structures makes them a possible candidate for mo recording. 


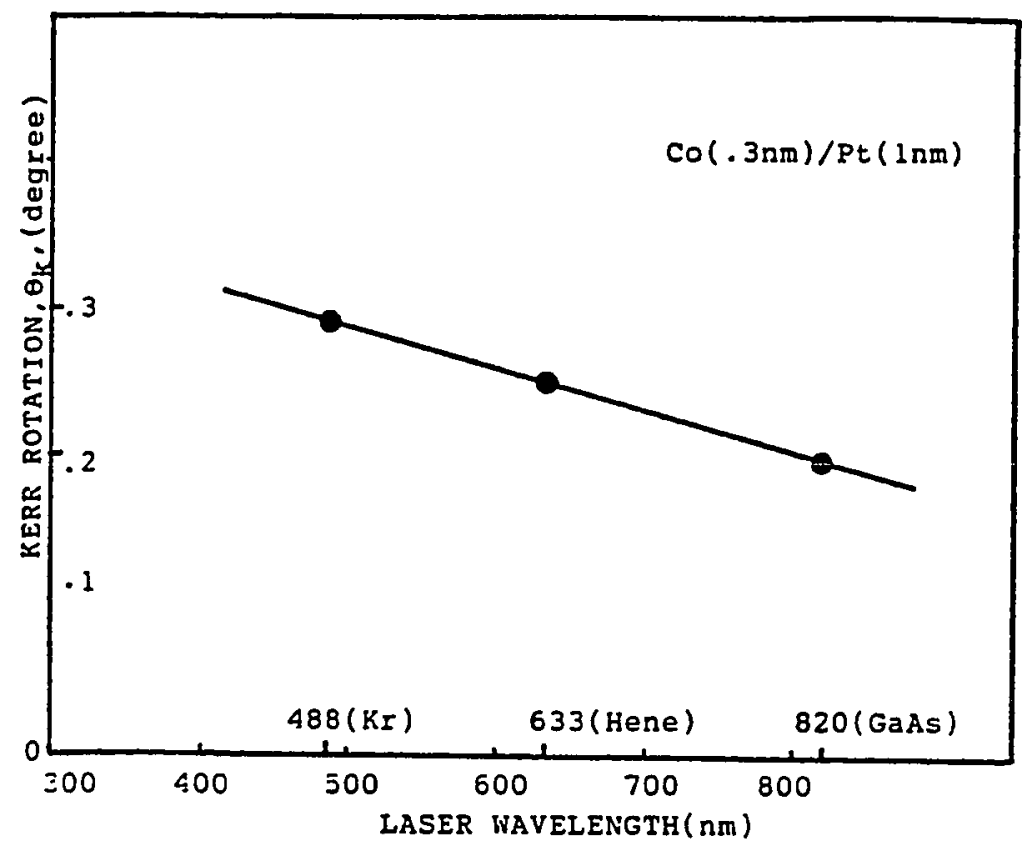

Figure 30. Kerr Rotation vs Reading Wavelength of a Co/Pt $0.3 / 1.0 \mathrm{~nm}$, $30 \mathrm{~nm}$ Thick Multilayer Film. 
Chapter 6

SUMMARY

Co/pt multilayer thin-films which are grown by e-beam evaporation onto 7059 corning glass substrates at room temperature have high perpendicular magnetic anisotropy and 1008 remanence when the thickness of the Pt layer is greater or equal to $1 \mathrm{~nm}$ and the co layer thickness is from 0.2 to $0.4 \mathrm{~nm}$. The Co $(0.3 \mathrm{~nm}) / \operatorname{Pt}(1 \mathrm{~nm})$ multilayer film has the highest coercivity $\left(\mathrm{H}_{\mathrm{C}}=1.9 \mathrm{KOe}\right), 100 \%$ remanence and a Kerr rotation, $\theta_{k}$, of about 0.2 degree. The Curie temperature of this film is $295^{\circ} \mathrm{C}$. By varying the Pt layer thickness both the Curie temperature and the Kerr rotation, which increase with increasing co/Pt ratio, can be selected.

The crystal structure of the film probably is the key parameter which decides whether the film has perpendicular or in-plane magnetization.

Compared to the currently prevailing RE-TM alloys, the Colpt multilayer structures have advantages in corrosion resistance, thermal stability, and enhanced kerr rotation at short wavelength. These attributes make Co/pt multilayer films a very promising candidate for MO recording. 
Chapter 7

REFERENCES

1. N. Inamura, "Characteristics of Magneto-optical Recording", J. Maq. Soc. Japan, Vol. 12, (1988) p.508.

2. F.O. Sequeda and H.P.D. Shied, "Optical Information storage Technology", IBM Research Report march 1990, p.28.

3. M. Chen and K.A. Rubin, "Progress of Erasable PhaseChange Materials", Proc. SPIE 1078, (1989) p.150.

4. N. Akahira, N. Yamaa, and M. Takao, "Recent Advances in Erasable Phase-Change Optical Disks", Proc. SPIE 899, (1988) P.188.

5. P. Argyres, "Theory of the Faraday and Kerr Effects in Ferromagnetics", Phys. Rev., Vol. 97, (1955) p.334.

6. P.S. Pershan, "Magneto-Optical Effects", J. Appl. Phys., Vol. 38, (1967) p.1482.

7. H.J. Williams, R.C. Sherwood, F.G. Foster, and E.M. Kelly, "Magnetic Writing on Thin Films of MnBi", $\mathrm{J}$. Appl. Phys., Vol. 28, (1957) p.1181.

8. P. Chandhari, J.J. Cuomo, and R.J. Grambino, "Amorphous Metallic Eilm for Buble Domain Applications", IBM. J. Res. Develop., Vol. 17, (1973) p.66.

9. C.F. Shelton, "Magneto-Optical Beam Addressable Storage Using MnGaGe and GdCo", IEEE Trans. Magn. MAG, Vol. 9, (1973) P.398.

10. M.H. Kryder, J. Appl Phys., Vol. 57, (1985) p.3913. 
H-P.D. Shied and M. Kryder, "The Influence of Deposition on the Magneto-Optic Effects in GdTbCo films", IEEE Trans. Magn. MAG, Vol. 21, (1985) p.1632.

11. E.E. Luborsky, "Kinetics for Changes in Anisotropy, Coercivity, and Argon Content of Transition Metal-Rare Earth film", J. Appl. Phys., Vol 57, (1985) p.3592.

12. D. Rugar, C.J. Iin, and R.H. Geiss, "Sub-Micron Domains for High Density Magneto-optic Data storage ( $2 \mathrm{u}$ Spacing)", IEEE Trans Maqn. MAG, Vol. 23, (Sept 1987) p.2263.

13. F. Sequeda, H. Do, and D.W. Chung, "The Effect of Sputter Deposition Conditions on Microstructure and Magnetic Propies of RE-TM Thin Films", International Conference of Metallurgical Coating, April 17-21, 1989, San Francisco, Raper C 3-3

14. J.C. Snots, R.H. Geiss, C.J. Iin, D. Rugar, and A. Bell, "Lorentz Microscopy of Micron-Sized laser written Magnetic Domains in TbFe", Appl Phys. Lett., Vol. 49, (1986) p.419.

15. M. Gomi, K. Utsuzi, and M. Abe, "RF Sputtered Films of Bi-Substituted Garnet for Magneto Optical Memory", IEEE Trans Magn. MAG., Vol. 22, (1956) p.1233.

16. M. Gomi, K. Satoh, and M. Abe, "Improvements in Optical and Magnetic Properties of Bi-substituted Garnet Sputtered Films for Magneto-Optical Recording", J. Appl. Phys., Vol. 63, (1988) p.3642.

17. T. Suzuki, F. Sequeda, H. Do, T. Huang, and G. Gorman, "Magnetic and magneto-optic properties of Bi-substituted garnet films crystallized by rapid thermal processing.", $34^{\text {th }}$ Annual Conference on Magnetisms and Magnetic Materials, November 28 - December 1, 1989, Boston, Paper AA-10.

18. P.G. Van Engen, K.H.J. Buschow, R. Jongebreur, and M. Erman, Appl. Phys Lett., Vol. 42, (1983) p.203. 
19. C. zenner, "Interaction between the d shells in the Transition Metals", Phys. Rev., Vol. 81, (1951) p.440.

20. P.F. Garcia, "Perpendicular magnetic anisotropy in Pd/Co and Pt/Co Thin Film Layered Structures", J.Appl. Phys., vol. 63. (1988) p.5066.

21. F.J.A. Den Broeder, D. Kuiper, A.P. Van de Mosselaer, and $w$. Hoving, "Perpendicular Magnetic Anisotropy of CoAn Multilayers Induced by Interface Sharpening", Phys. Rev. Lett., Vol. 60, (1988) p.2769.

22. H.P.D. Shied, "Magneto-optical Recording MediaFabrication, Characterization and Optimization", Ph.D Thesis, Carnegie Mellon University, (1986) P.16.

23. W.H. Meikle John, "Magneto Optics: A Thermomagnetic Recording Technology", Eroceedings of the IEEE, Vol. 74, (1986) p.11.

24.G.E. Thomas, "Future Trends in Optical Recorting", Philips. Tech. Rev., Vol. 44, (1988) p.51.

25. L.J. Maissel and R. Glang, "Handbook of Thin Film Technology", Mac. Graw-Hill, Newyork (1986) p.8:32.

26.R.W. Vook, "Thin Film Growth", Mat. Res. Soc. Symp. Proc., Vol. 103, (1988) p.3.

27. F.C. Frank, and J. .H. Van der Merwe, "One-Dimentional Dislocations. II. Misfitting Monolayers and Oriented Overgrowth", Proc. Roy. Soc., Series A, Vol. 198 (1949) p.217.

28. J.C. Bean, "Formation and structure of Fe/Cu(001) Interfaces, Sandwitches and Superlattices", J.Vac. Sci. Tech., Vol. A2, (1984) p.436.

29. LL. Hinchey, and D.L. Mills, J. Appl. Phys,, Vol. 57, (1985) p.3687.

30.D. Treves, "Platinum-Cobalt films for Digital MagnetoOptic Recording", J. Appl. Phys., Vol. 46, (1975). 
31. J. A. Aboaf, S.R. Herd, and E. Klokholm, "Magnetic Properties and Structure of Cobalt-Patinum Thin Films", IEEE Trans. Magn. MAG-19, (1983) p.1514.

32. N. Sato, "Crystallographic Structure and Magnetism of Co-Pd and Co-Pt Films with and Artificially Layered Structure", J.Appl. Ehys., Vol. 64, (1988) p.11.

33.W.B. Zeper, F.J.A.M. Greidanus, and P.E. Carcia, "Evaporated Co/pt Layered Structures for Magneto-Optical Recording", Research Manuscript Rhilips Research Laboratory Netherlands 1989, (Intermaq'89, 3/89, Washington DC, Paper 58-09)

34. Leroy L. Chang and B.C. Giessen, "Synthetic Modulated Structures", Academic Press inc., (1985) p.43-73. 